\title{
Detection of complete and partial chromosome gains and losses by comparative genomic in situ hybridization
}

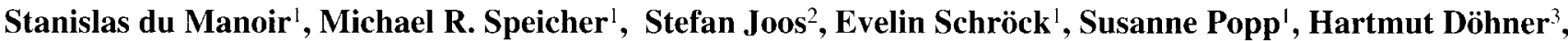 \\ Gyula Kovacs $^{4}$, Michel Robert-Nicoud ${ }^{5}$, Peter Lichter ${ }^{2}$, Thomas Cremer \\ ' Institut für Humangenetik, Im Neuenheimer Feld 328, W-6900 Heidelberg, Germany \\ ? Angewandte Tumorvirologie, Deutsches Krebsforschungszentrum, Im Neuenheimer Feld 280. W-6900 Heidelberg, Germany \\ ${ }^{3}$ Medizinische Klinik und Poliklinik V, Universität Heidelberg, Hospitalstr. 3, W-6900 Heidelberg, Germany \\ ${ }^{4}$ Imperial Cancer Research Fund Laboratories, P.O. Box 123, Lincoln's Inn Fields, London WC2A 3PX. UK \\ ${ }^{5}$ Equipe de Reconnaissance de Formes et de Microscopie Quantitative. TIM3, USR CNRS B00690. CERMO, Université Joseph Fourier, \\ Grenoble, France
}

Received: 30 October 1992

\begin{abstract}
Comparative genomic in situ hybridization (CGH) provides a new possibility for searching genomes for imbalanced genetic material. Labeled genomic test DNA, prepared from clinical or tumor specimens, is mixed with differently labeled control DNA prepared from cells with normal chromosome complements. The mixed probe is used for chromosomal in situ suppression (CISS) hybridization to normal metaphase spreads (CGH-metaphase spreads). Hybridized test and control DNA sequences are detected via different fluorochromes, e.g., fluorescein isothiocyanate (FITC) and tetraethylrhodamine isothiocyanate (TRITC). The ratios of FITC/TRITC fluorescence intensities for each chromosome or chromosome segment should then reflect its relative copy number in the test genome compared with the control genome, e.g., 0.5 for monosomies, 1 for disomies, 1.5 for trisomies, etc. Initially, model experiments were designed to test the accuracy of fluorescence ratio measurements on single chromosomes. DNAs from up to five human chromosome-specific plasmid libraries were labeled with biotin and digoxigenin in different hapten proportions. Probe mixtures were used for CISS hybridization to normal human metaphase spreads and detected with FITC and TRITC. An epifluorescence microscope equipped with a cooled charge coupled device (CCD) camera was used for image acquisition. Procedures for fluorescence ratio measurements were developed on the basis of commercial image analysis software. For hapten ratios $4 / 1,1 / 1$ and $1 / 4$, fluorescence ratio values measured for individual chromosomes could be used as a single reliable parameter for chromosome identification. Our findings indicate (1) a tight correlation of fluorescence ratio values with hapten ratios, and (2) the potential of fluorescence ratio measurements for multiple color chromosome painting. Subsequently, genomic test DNAs, prepared from a
\end{abstract}

Correspondence to: T. Cremer patient with Down syndrome, from blood of a patient with Tcell prolymphocytic leukemia, and from cultured cells of a renal papillary carcinoma cell line, were applied in $\mathrm{CGH}$ experiments. As expected, significant differences in the fluorescence ratios could be measured for chromosome types present in different copy numbers in these test genomes, including a trisomy of chromosome 21 , the smallest autosome of the human complement. In addition, chromosome material involved in partial gains and losses of the different tumors could be mapped to their normal chromosome counterparts in $\mathrm{CGH}$-metaphase spreads. An alternative and simpler evaluation procedure based on visual inspection of CCD images of $\mathrm{CGH}$-metaphase spreads also yielded consistent results from several independent observers. Pitfalls, methodological improvements, and potential applications of CGH analyses are discussed.

\section{Introduction}

Gains and losses of whole chromosomes or chromosomal segments have been observed in many malignant tumors. They also constitute a major cause of mental retardation and malformation syndromes. The possibilities of detecting and precisely defining such genetic imbalances are still limited in spite of the important advances of classical and molecular cytogenetics. The development of chromosome banding some 25 years ago (Caspersson et al. 1968) has provided an efficient tool for the comprehensive analysis of chromosome complements, but has often been hampered by difficulties in preparing high quality metaphase chromosome spreads from clinical and tumor cell samples, particularly in the case of solid tumors. Even with optimally banded chromosomes, cytogeneticists may not be able to determine the origin of 
marker chromosomes in complex rearrangements. With an incomplete karyotype to hand, it is impossible to decide, with confidence, which chromosome segments are genetically balanced and which are not.

The rapid development of non-isotopic in situ hybridization techniques and the generation of numerous chromosome-specific DNA probes have provided new possibilities for complementing chromosome banding techniques (for a review, see Lichter et al. 1991). Interphase cytogenetics has allowed the assessment of numerical and structural chromosome aberrations directly in the cell nucleus (for reviews, see Lichter et al. 1991; Poddighe et al. 1992). Recently, multiple color fluorescence in situ hybridization (FISH) has further enhanced our capacity for identifying chromosome aberrations with speed and accuracy (Nederlof et al. 1990; Nederlof 1991; Ried et al. 1992). However, in order to select DNA probes useful for the detailed analysis of a clinical or tumor cell sample, previous knowledge of the types of expected aberrations is required.

Molecular genetics has provided additional powerful tools for use in the search for genetic imbalances in genomic DNA. The consistent loss of maternally or paternally derived chromosome segments in a tumor cell population can be detected by concomitant losses of heterozygosity of alleles of DNA markers (Bishop 1987). This approach requires large numbers of polymorphic DNA markers informative for the patient in question. Although amplifications of specific DNA sequences can easily be detected, e.g., in Southern blots from tumor DNA, it might be a problem to choose the appropriate probes. It is even more difficult to distinguish between two and three copies of a DNA segment. The global analysis of genomic DNA from tumor samples for chromosomal gains and losses by presently available methods of molecular genetics therefore remains too laborious to be implemented in routine diagnostic schemes.

The limitations of the methods mentioned above have caused an urgent need for new methods to allow the rapid and comprehensive assessment of cells for genetic imbalances in cases where genomic DNA is the only material available. In this study, we describe and test a new approach, termed comparative genomic in situ hybridization (CGH). The first experimental demonstration of $\mathrm{CGH}$ was presented by Kallionieni et al. (1992). For CGH, genomic test DNA prepared from clinical or tumor specimens (further referred to as test genomes) is chemically modified with certain haptens (e.g., with biotin). Genomic control DNA prepared from cells with normal chromosome complements (further referred to as control genomes) is labeled with a different hapten (e.g., digoxigenin). Test and control DNAs are then mixed in defined proportions, e.g., $1: 1$, and used as a probe for chromosomal in situ suppression (CISS) hybridization (Lichter et al. 1988; Pinkel et al. 1988) on metaphase spreads with normal chromosome complements $(46, \mathrm{XY}$ or $46, \mathrm{XX}$; further referred to as CGH-metaphase spreads). In such an experiment, homologous chromosome-specific DNA sequences, present in both test and control genomic DNAs, compete for the same target chromosomes. Hybridized test and control DNA sequences are detected by different fluorochromes, e.g., fluorescein isothiocyanate (FITC) and tetraethylrhodamine isothiocyanate (TRITC), respectively. The resulting ratios of FITC/TRITC fluorescence intensities for each chromosome should reflect the relative copy numbers of the homologous sequences contained in the two genomic DNAs. The fluorescence ratio obtained for chromosomes disomic in the test and the control genome should decrease by a factor of 0.5 for monosomies, and become zero for nullosomies. It should increase by a factor of 1.5 for trisomies, by a factor of 2 for tetrasomies, 2.5 for pentasomies, and so forth. Whereas an internal standard provided by the simultaneous CISS hybridization of control DNA is preferable for detecting small differences of copy numbers between test and control genomes, genomic tumor DNA is sufficient as a probe for CISS hybridization to normal metaphase chromosome spreads in order to map sequences present in large copy numbers in homogeneously stained regions or double minutes (see Joos et al. 1992).

In order to implement and test such a strategy, we have tried to answer the following questions. Firstly, is it possible to measure fluorescence ratio (FR) values for individual chromosomes with the accuracy demanded for successful CGH experiments? To answer this question, a series of individual chromosomes was painted with two fluorochromes in different proportions. These experiments were also designed to determine whether individual chromosomes could be identified solely on the basis of FR measurements. Secondly, is it possible to identify gains and losses of large, medium, and small-sized chromosomes by $\mathrm{CGH}$, and can such a diagnosis be reliably obtained, even in cases where numerous numerical abnormalities have occurred in certain tumor genomes? Thirdly, is it also possible to identify partial chromosomal gains and losses by CGH and, if so, can one define the breakpoints for marker chromosomes containing unbalanced chromosomal segments?

\section{Materials and methods}

\section{Cells}

The cell line ACHN was established from a papillary renal cell carcinoma, and has been karyotyped previously (Kovacs et al. 1991). Peripheral blood from an untreated patient with $\mathrm{T}$-cell prolymphocytic leukemia (T-PLL) was obtained at the time of diagnosis. By immunophenotyping, $85 \%$ of the leucocytes were CD4-positive and $88 \%$ $\mathrm{CD} 7$-positive, reflecting the high portion of T-cell prolymphocytes. Blood cells were cultivated in the presence of interleukin 2, and metaphase spreads were prepared using standard procedures.

\section{CGH-metaphase spreads}

Metaphase spreads for CGH experiments were prepared from phytohemagglutinin (PHA)-stimulated lymphocytes of healthy male individuals $(46, X Y)$ using standard procedures of hypotonic treatment and methanol/acetic acid fixation $(3: 1, \mathrm{v} / \mathrm{v})$.

\section{Labeling schemes for chromosome-specific DNA libraries with different hapten ratios (biotin/digoxigenin)}

DNA prepared from pBS-libraries constructed from flow-sorted human chromosomes $1,4,8,13$ and 16 (Collins et al. 1991), kindly provided by Joe Gray (University of California, San Francisco, CA), was nick-translated using digoxigenin-11-dUTP (Boehringer Mannheim) and/or biotin-11-dUTP (Sigma) as haptens (Lichter and Cremer 1992). Modified nucleotides were incorporated in various proportions into library DNAs by adding mixtures of digoxigenin-11-dUTP and biotin-11-dUTP to the 
nick-translation assay $(64 / 1,16 / 1,4 / 1,1 / 1,1 / 4,1 / 16,1 / 64)$. For each assay, the final concentration of digoxigenin-11-dUTP plus biotin-11dUTP was adjusted to $0.04 \mathrm{~m} M$. The final concentrations for other nuclcotides were $0.05 \mathrm{~m} M$ dATP. $0.05 \mathrm{~m} M$ dCTP, $0.05 \mathrm{~m} M$ dGTP, $0.01 \mathrm{~m} M$ dTTP.

\section{DNA probes and labeling procedures for $C G H$ experiments}

Control genomic DNAs were prepared from blood of a healthy male $(46, X Y)$ or from human placenta $(46, X X)$. Test genomic DNAs were extracted from the cell line ACHN, from peripheral blood of a T-PLL patient, and from peripheral blood of a child with Down syndrome $(47, X X,+21)$. Using standard nick-translation procedures (sce above). control DNA and test DNA were labeled with digoxigenin-1 I-dUTP or biotin-11-dUTP. The concentrations of control and test DNAs were measured. and I: 1 mixtures of differently labeled test and control genomic DNAs were prepared.

\section{CISS hybridization and probe detection}

CISS hybridization and probe detection were carried out as described in detail by Lichter and Cremer (1992) with the following modifications. In experiments with chromosome-specific DNA libraries and for each slide (area $18 \times 18 \mathrm{~mm}$ ), a total of $300 \mathrm{ng}$ labeled library DNA for each

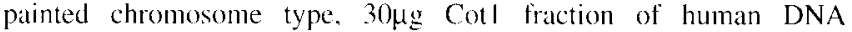
(BRL/Life Technologies) and 30 $\mu \mathrm{g}$ sonicated salmon testes DNA (Sigma) were combined, ethanol precipited and resuspended in IOpl hybridization mixture containing $50 \%$ formamide, $10 \%$ dextran sulfate in $2 \times$ SSC: $0.3 \mathrm{M} \mathrm{NaCl}, 30 \mathrm{mM}$ Na citrate. $\mathrm{pH} 7.0$. After CISS hybridization to $\mathrm{CGH}$-metaphase spreads and post-hybridization washes, the biotinylated probes were detected using avidin conjugated to TRITC (Vector Laboratories). One round of signal amplification was performed as described by Pinkel en al. (1986). Digoxigenin-labeled probes were detected by indirect immunolluorescence using mouse anti-digoxin antibodies (Sigma) and FITC-conjugated sheep anti-mouse antibodies (Sigma). In other experiments, avidin conjugated to AMCA (aminomethyl-coumarin acetic acid, Vector Laboratories) or FITC (Vector Laboratories) was used in combination with TRITC-conjugated sheep anti-mouse antibodies. No counterstaining was applied.

In CGH experiments, $2 \mu \mathrm{g}$ of a $1: 1$ mixture of differently labeled test DNAs and control DNAs was used per slide in combination with 50-300 $\mu \mathrm{g}$ unlabeled Cotl DNA. CISS hybridization to CGHmetaphase spreads was carried out for $1-3$ days. For control $\mathrm{CGH}$ experiments, a $1: 1$ mixture made from differently labeled aliquots of control DNA (46,XY) was used. Post-hybridization washes were carried out to a stringency of $0.1 \times \mathrm{SSC}$ at $60^{\circ} \mathrm{C}$. Biotin- and digoxigenin-labeled sequences were visualized as described above via FITC and TRITC or vice versa. DAPI (4.6-diamidino-2-phenylindole $2 \mathrm{HCl}$ ) was used as the only counterstain. The best results for DAPI banding were achieved when DAPI stock solution (Serva No. 18860) was diluted 1:20000 in $4 \times \mathrm{SSC} / 0.2 \%$ Tween and applied for $3 \mathrm{~min}$. Slides were mounted in fluorescence-antifading buffer.

\section{Fluorescence microscopy}

For epifluorescence microscopy, a Zeiss Axiophot microscope equipped with a $100 \mathrm{~W}$ lamp was used with the following filter sets: No. 10 (BP 450-490), FT 510, BP 515-565) for FITC signals, No. 15 (BP 546, FT 580, LP 590) for TRITC signals, No. 01 (BP 365, FT 395, LP 397) for DAPI fluorescence, and a new filter set (BP 365, FT 395, BP 450-490) for AMCA signals. Filter sets were specially aligned by the Carl Zeiss company to minimize image shifts. All images were taken via the PlanNEOFLUAR $63 x / 1.25$ oil immersion lens. Microphotographs were taken with Agfachrome 1000 RS color-slide films. Photographs from the screen were taken with 50 or 100 ASA films.

\section{Image acquisition}

Digital fluorescence images were recorded using a cooled CCD (charge coupled device) camera (Photometrics. Tucson, AZ, USA) with the Kodak KAF 1400 chip $(1317 \times 1035$ pixels $)$. The mercury lamp was carefully centered in order to achieve the best possible homogeneity of the illumination field. The field diaphragm was closed to the limit of the camera image field. A selected area of $512 \times 512$ pixels was adjusted to the optical center of the microscope field and used for image recording. A gray level image was taken separately for each fluorochrome using the appropriate filter sets and the software package Nu200 2.0 (Photometrics) implemented on a Macintosh Quadra 900. Each image was stored under the TIFF-format. The optimal exposure time for each slide and filter set was chosen in order to avoid saturation values in all pixels, and to cover at least half the total dynamic range of the camera. Exposure times and all optical settings of the microscope were kept constant for a whole series of image acquisitions. Identification of chromosomes was made on the basis of CCD images following DAPI staining.

\section{Image processing}

Digital images were processed either by the SAMBA 2005 image analyzer system (Alcatel-TITN. Grenoble, France) or the TCL Image software (Multihouse. Amsterdam, The Netherlands). To correct for small geometric shifts. one chromosome was selected and segmented in all CCD images acquired from a given metaphase spread. The positions of the gravity centers of the segmented masks obtained for this chromosome were used to align the images recorded for the different fluorochromes (adapted from Waggoner et al. 1989).

FR measurements in CISS hybridization experiments with chromosomespecific DNA libraries. CCD images were acquired for the two fluorochromes applied in a given experiment (e.g., FITC/TRITC, AMCA/TRITC or AMCA/FITC). For each image, segmentation of the painted chromosomes was obtained by adaptative thresholding based on the gray level histogram (Usson et al. 1987). An "OR logical procedure" was applied to the two segmentation mask images to obtain the final segmentation mask image containing the masks for all chromosomes painted with either one fluorochrome or both fluorochromes. For each painted chromosome, two integrated fluorescence values, [e.g., IF(FITC) and IF(TRITC)], were obtained by summing the gray level values for each pixel of a given mask. These integrated fluorescence values were divided by the mask area to calculate the fluorescence intensity values F(FITC) and F(TRITC) for each painted chromosome. The background fluorescence intensity $(\mathrm{Fb})$ was determined from a segmentation mask defined for all non-painted chromosomes. The corrected fluorescence intensities Fcor(FITC) and Fcor(TRITC) for each painted chromosome were calculated by subtracting $\mathrm{Fb}(\mathrm{FITC})$ or Fb(TRITC) from the relevant $F$, i.e., Fcor $=F-F b$. The FR for each painted chromosome was obtained by dividing the corrected fluorescence intensities Fcor(FITC)/Fcor(TRITC).

Finally, for each chromosome type, the means of the Fcor and FR values obtained for individual chromosomes in a series of metaphase spreads, $F(\circ) r$ and $F R$, were calculated.

$F R$ measurements in CGH experiments. Three CCD images were acquired using specific filter-sets for DAPI, FITC and TRITC. Image shifts were corrected as described above. After a local contrast procedure (TCL-lmage software. Multihouse), the three images were segmented separately to generate three "intermediate I" segmentation mask images. An "AND logical procedure" was applied to the "intermediate I" segmentation mask image. This image combines the masks that are present in each of the three "intermediate I" segmentation mask images. The final segmentation mask image was obtained after interactive separation of overlapping chromosomes in the "intermediate 2" segmentation mask image. F(FITC) and F(TRITC) values were determined for each chromosome as described above. The background fluorescence intensity $(\mathrm{Fb})$ was defined for each $\mathrm{CCD}$ image as the fluorescence intensity of the area outside individual chromosome masks in the "intermediate 2" segmentation mask image. Fcor(FITC), Fcor(TRITC) and $\mathrm{FR}$ values for each individual chromosome, as well as Fcor(FITC), $F(O)$ (FITC) and $F R$ values for a population of a given chromosome type (chromosome 1,2, etc.) evaluated in a series of metaphase spreads, were determined as described above.

Evaluation of chromosome imbalances based on fluorescence ratio measurements in CGH experiments. In an attempt to define an empirical threshold for the identification of chromosomal gains and losses in CGH experiments, control CISS hybridization experiments were carried out using a 1:1 mixture of biotin- and digoxigenin-labeled control DNA $(46, X Y)$ detected with FITC and TRITC, respectively. In three metaphase spreads, fluorescence ratios were determined for each chro- 
mosome. For this chromosome population, the mean of the $\log (\mathrm{FR})$ values and the $95 \%$ confidence interval was calculated (mean \pm 1.96 times the SDM). The limits of the $\log (F R)$ confidence interval were converted back to FR values. Note that using this procedure, the upper limit of the confidence interval is equal to reciprocal values of the lower limit (1/lower limit). In CGH experiments, chromosome types with an $F R$ outside of this range were considered to be over- or under-represented in the test genome.

$F R$ images. The FITC image of a $\mathrm{CGH}$-metaphase spread as recorded by $C C D$ was divided (pixel by pixel) by the TRITC image as recorded by $\mathrm{CCD}$, and normalized by multiplication with a factor $A[A=$ IF(TRITC)/IF(FITC), where IF(FITC) and IF(TRITC) are defined as the integrated fluorescence values for the combined mask areas of the "intermediate 2 " segmentation mask image, as given above]. The logarithm of the normalized value from each pixel was calculated to produce a $\log$ (ratio) image. For visualization, 256 gray levels were used, and the mean of the $\log$ (ratio) image values as determined inside the combined masks of the "intermediate 2" segmentation mask image was set to gray level 128. Using a three-color look-up table, pixels with a gray level above the upper threshold defined from control CGH experiments (see above) are presented in green, pixels with gray levels below the lower threshold are presented in red, and pixels with gray levels within the threshold-range are presented in blue.

Evaluation of chromosome imbalances based on visual examination of $C C D$ images from CGH-metaphase spreads. Several observers, who were not informed of the clinical and cytogenetic diagnoses of the two tumors studied by $\mathrm{CGH}$, were instructed to perform a visual side-byside comparison of FITC and TRITC images of CGH-metaphase spreads, as recorded by $C C D$, in the following way. (1) To examine the CCD image from the fluorochrome used for the detection of hybridized test DNA and to consider chromosomes that appear either considerably more intensely or considerably less intensely painted that the majority of the chromosomes, and therefore are considered to be suspicious for gains or losses in the test genome. (2) To examine the corresponding CCD image obtained from the hybridized control DNA and to mark as candidates for a gain or loss in the test genome only those of the suspi- cious chromosomes considered above, showing a normal hybridization intensity in the control CCD image. Chromosomes presenting a correspondingly higher or lower fluorescence intensity in both CCD images should be considered as being balanced in the test genome.

Care was taken that each observer performed the evaluation independently without any interference from the experimentors. Observers were allowed to change the brightness of the images to facilitate the evaluation of relative painting intensities of different chromosomes. DAPI images were not available to them, to ensure that their evaluation procedure was based solely on the visual assessment of painting intensities. A threshold frequency for random assignments of marked chromosomes was calculated for each observer separately by dividing the total number of marked chromosomes by 24 , the number of chromosome types contained in male CGH-metaphase spreads. In cases where the chromosome arms were considered separately, the denominator was changed accordingly. Thresholds were calculated independently for chromosome gains and losses, since the evaluation of CGH-metaphase spreads in control experiments with differently labeled normal genomic DNA showed that observers more often indicated a possible gain than a possible loss of a chromosome (see Results; Fig. 11, bottom). Chromosome types that were marked above threshold frequencies were considered as candidates for over-representation and under-representatioin, respectively, in the test genome.

\section{Results}

\section{Identification of chromosomes painted with two fluorochromes by FR measurements}

A reliable procedure for the identification of chromosomes based on two-color chromosome painting and FR measurements should have the following characteristics. (1) Mixtures of chromosome-specific library DNAs with distinctly different hapten ratios (e.g., biotin/digoxigenin) have to be pre-

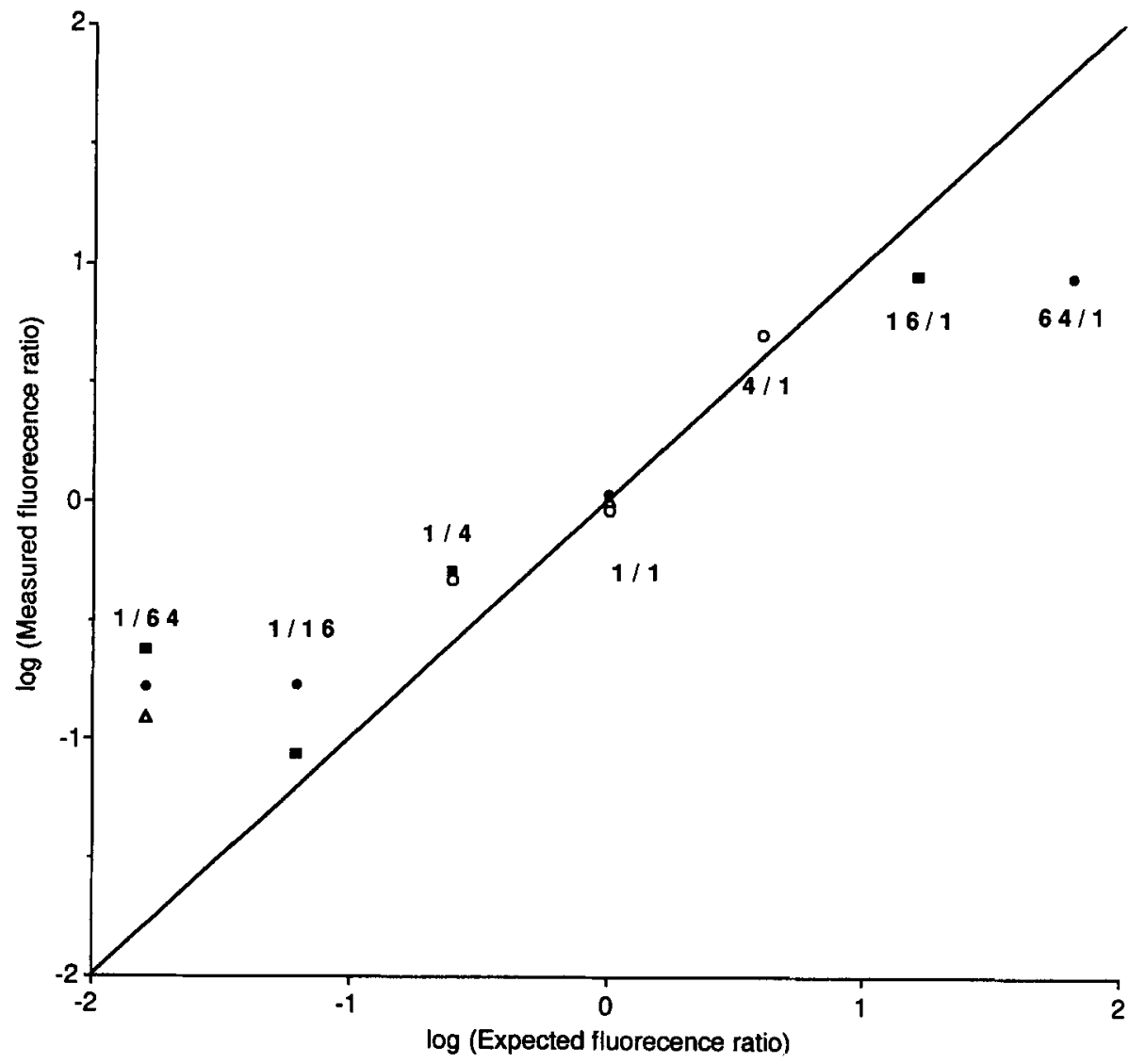

Fig. 1. Measured versus expected $F R$ of painted chromosomes. This experiment demonstrates a range of proportionality between hapten ratios (biotin-11-dUTP and digoxigenin-11-dUTP) applied in nick-translation assays of chromosome-specific DNA libraries and the means of FITC/TRITC fluorescence ratios $(F R)$ measured for painted chromosome types. Chromosome-specific library DNAs, labeled with biotin-11-dUTP and digoxigenin-11-dUTP in various proportions as indicated, were used to paint chromosomes $4,8,13$ and 16 in normal human metaphase spreads $(46, X Y)$. $F R$ values were determined for each chromosome type in four independent experiments. Each point represents the $F R$ value from 30 chromosomes. The logarithm of $F R$ (ordinate) is compared with the logarithm of the mean fluorescence values expected on the basis of the hapten ratios. A proportionality between measured and expected log-values is found for hapten ratios from $1 / 4$ to $4 / 1$. Note that this proportionality was obtained in experiments using different batches of nucleotide mixtures and that different chromosome types labeled with the same hapten ratio show similar FR values. $\triangle$ Chromosome 4 , - Chromosome 8, Chromosome 13, O Chromosome 16 
pared with high accuracy and reproducibility. (2) Chromosome-specific library DNAs from different chromosomes labeled with the same hapten ratio should yield the same $F R$ values for each chromosome type (e.g., 1, 2 etc). (3) DNA probes for different chromosomes labeled with different hapten ratios should yield a range of $\mathrm{FR}$ values for individual chromosomes of each type evaluated in a series of metaphase spreads that does not overlap with the ranges obtained for the other chromosome types.

In preliminary experiments, chromosome-specific library DNAs for chromosomes 4, 8, 13 and 16 were nick-translated separately with either biotin or digoxigenin, and mixed thereafter in various proportions. The accuracy with which such mixtures could be produced for individual chromosomes in
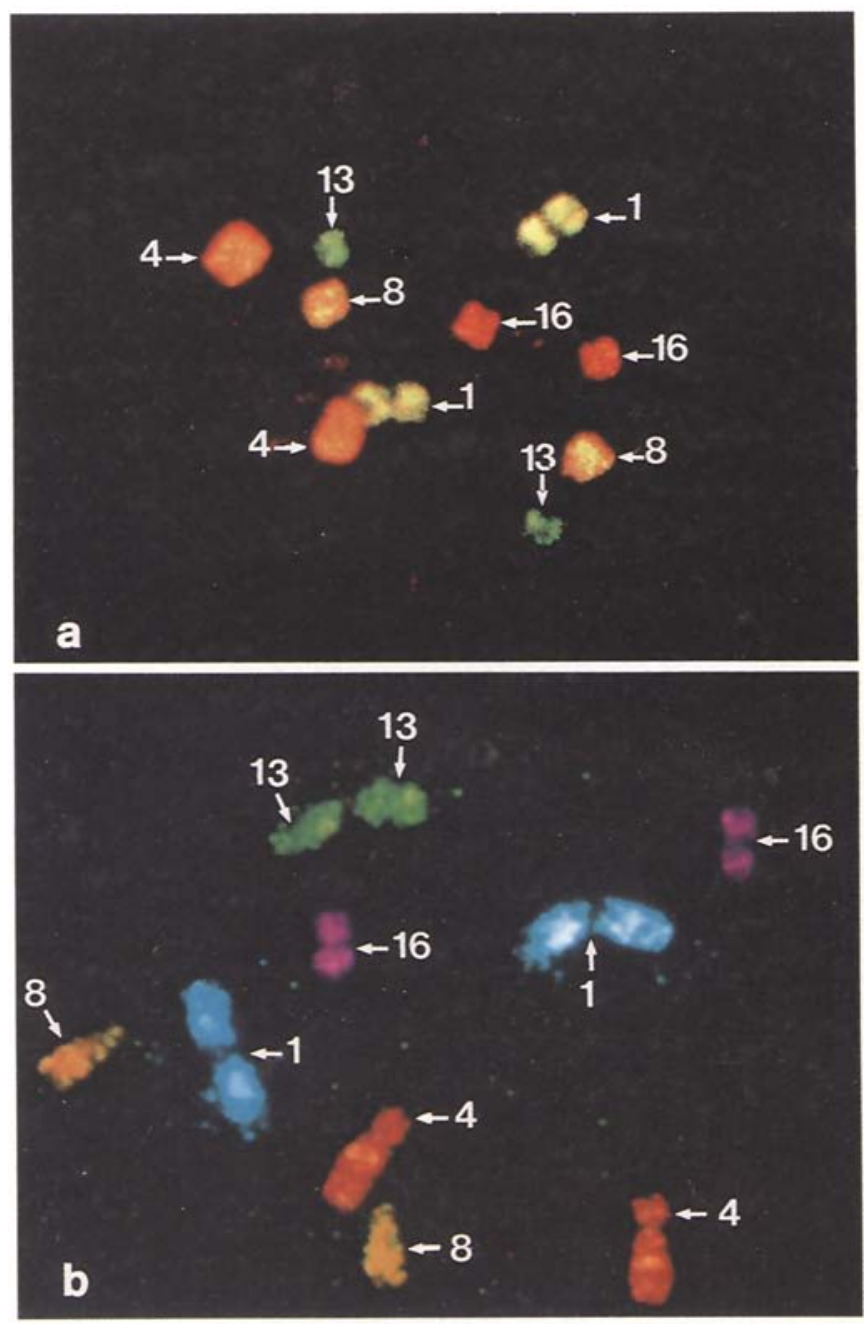

Fig. 2a, b. CISS-hybridization to normal human metaphase spreads (46,XY) performed with DNA libraries specific for chromosomes 1, 4, 8,13 and 16 , labeled with various hapten proportions (biotin/digoxigenin) and detected with FITC/TRITC. a Micrograph of a typical metaphase obtained by sequential exposure of a color-slide film with FITC- and TRITC-specific filter combinations. Note that all chromosome types can be distinguished by natural fluorescence colors. $\mathrm{b} C \mathrm{CD}$ image of another metaphase spread. A continuous pseudocolor look-up table was chosen to display the range of individual fluorescence ratio values obtained for painted chromosomes in 14 metaphase spreads (compare Fig. 3). Note that the color for each chromosome type is different, whereas the two homologs of each chromosome type display the same color repeated experiments, however, was not satisfactory in our hands. To overcome this problem in further experiments, biotin-11-dUTP and digoxigenin-1 1-dUTP were first mixed in various proportions $(64 / 1,16 / 1,4 / 11 / 1,1 / 4,1 / 16,1 / 64)$ and used in nick-translation assays to label chromosome-specific library DNAs for chromosomes 1, 4, 8, 13 and 16 simultaneously with the two haptens. Detection of biotin and digoxigenin was achieved with TRITC and FITC, respectively. The latter procedure yielded highly reproducible $F R$ values measured with a cooled CCD camera in a series of CISS hybridization experiments including probes labeled with several independently produced batches of nucleotide mixtures (Fig. 1). The observed $F R$ values were directly proportional to the chosen hapten ratios of biotin-11-dUTP/digoxigenin11-dUTP at least within the range from $4 / 1$ to $1 / 4$, whereas $F R$ values measured for hapten ratios chosen outside this range showed a considerable deviation from expected values.

To test whether individual chromosomes can be discriminated on the basis of FRs, a CISS hybridization experiment was carried out with chromosome specific libraries for chromosomes $1,4,8,13$ and 16 . The following hapten ratios (biotin/digoxigenin) were chosen: chromosome 1 (4/1), chro-

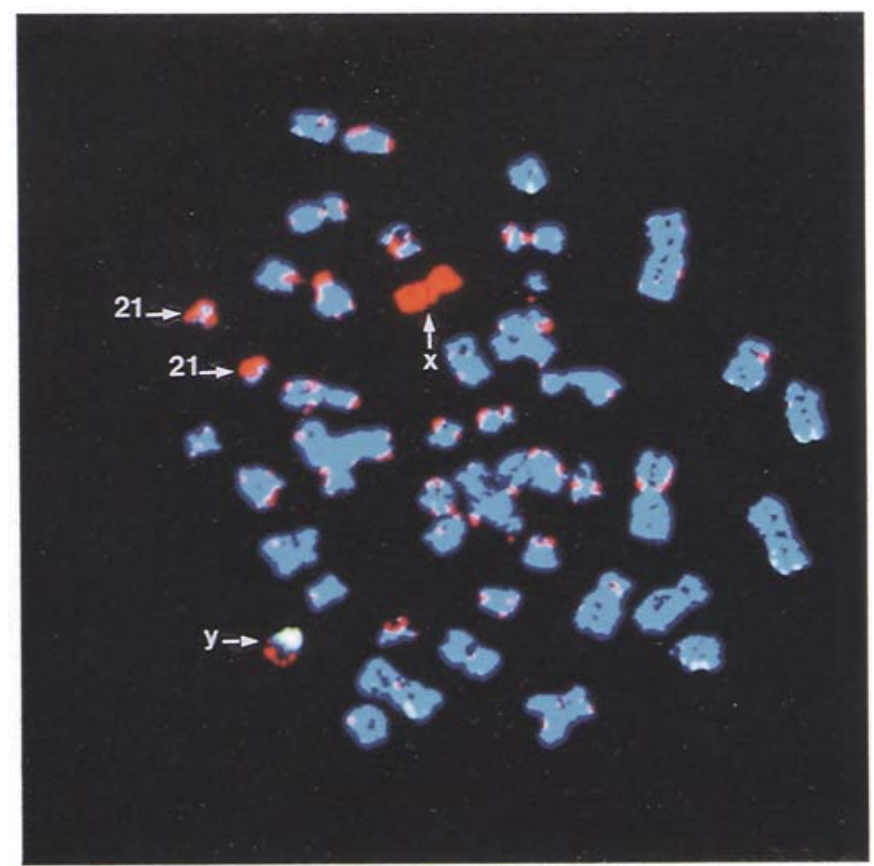

Fig. 4. FR image of a CGH-metaphase spread hybridized with $(47, X X,+21)$-test DNA detected with TRITC, and $(46, X Y)$-control DNA detected with FITC. A three-color look-up table was used for a pixel by pixel display of FR values obtained with FITC/TRITC. Blue in dicates a range of ratio values obtained for chromosomes represented in equal numbers in both the test and the control genome; red indicates ratios suggestive of chromosomes present in higher numbers in the test genome; green indicates ratios suggestive of lower numbers in the test genome. Scattered red and green spots along some of the chromosomes are methodological artefacts and show varying localization in different $\mathrm{CGH}$-metaphase spread. The X chromosome and both chromosomes 21 are consistently colored red in this and other $\mathrm{CGH}$-metaphase spreads. On the Y chromosome, the euchromatin part is consistently colored green indicating the presence of $Y$-chromosome-specific sequences in the control genome but not in the test genome (except for $X-Y$ homolog sequences). Painting of the heterochromatic part of the $Y$ was suppressed by Cot 1 DNA. Red dots seen on this heterochromatic region are artefacts 


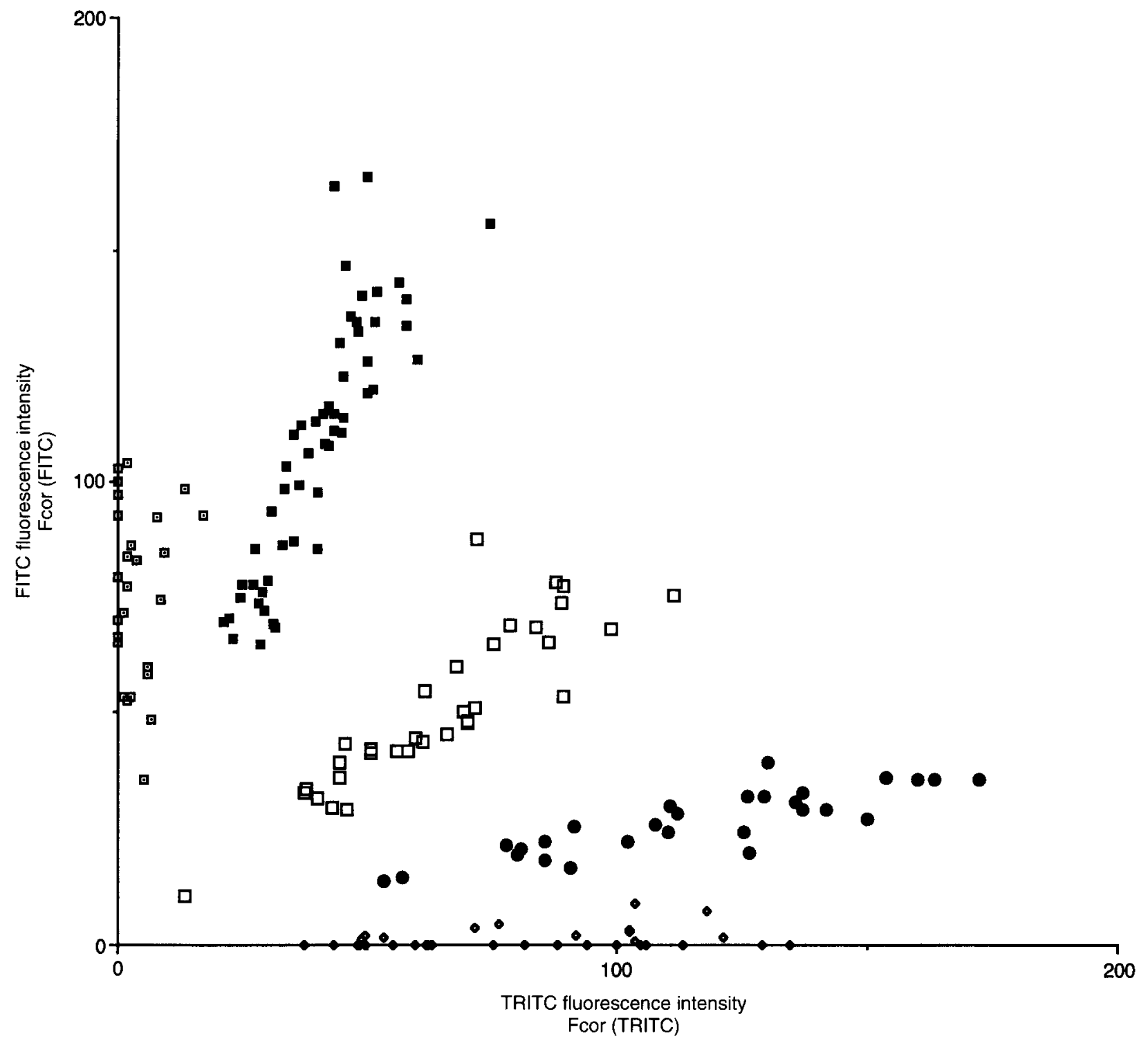

Fig. 3. FITC fluorescence intensities versus TRITC fluorescence intensities of painted chromosomes [Fcor(FITC) vs Fcor(TRITC)]. Chromosomes $1,4,8,13$ and 16 were painted with various proportions of FITC and TRITC. Each point represents the fluorescence intensity measured for an individual chromosome mask obtained in 14 metaphase spreads. The number of masks obtained for some chromosomes is greater than 28 since, because of the lack of painting of centromeric or paracentromeric heterochromatin (compare Fig. 2), separate masks were sometimes obtained for each chromosome arm. Note that the ranges of Fcor(FITC) and Fcor(TRITC) values obtained for each chromosome type show considerable overlap; this is not the case for the range of FR values $(\mathrm{FR}=\mathrm{Fcor}(\mathrm{FITC}) / \mathrm{Fcor}$ (TRITC)). Accordingly, each individual painted chromosome can be identified by this criterion (compare Fig. 2b). $\square$ chromosome 1, chromosome $4, \square$ chromosome $8, \square$ chromosome $13, \uparrow$ chromosome 16

mosome $4(1 / 4)$, chromosome $8(1 / 1)$, chromosome 13 (biotin only), chromosome 16 (digoxigenin only). Figure $2 \mathrm{a}$ shows a color microphotograph from a typical metaphase chromosome spread with five pairs of differently colored chromosome types after double exposure of TRITC and FITC fluorescence. Fourteen metaphase spreads with various condensation state of the chromosome complements were an- alyzed in detail by FR measurements. The range of FR values observed in this data set for each painted chromosome type did not overlap with the range of FR ratios obtained for any other painted chromosome type (Fig. 3). Accordingly, a continuous pseudocolor look-up table could be chosen to identify unequivocally a given chromosome type by color (Fig. 2b).

The above results demonstrate that individual FR values can be used as a single reliable parameter to identify chromosomes independently of their condensation states. From the data presented in Fig. 3, we predict that in CGH experiments the range of FRs (test DNA/control DNA) obtained for chromosome monosomy (expected value 0.5), disomy (expected value 1), trisomy (expected value 1.5) and tetrasomy (expected value 2 ) should show considerable overlap.

\section{Detection of differences in sex chromosome constitution and of trisomy 21 by $\mathrm{CGH}$ with $(47, X X+21)$ test $D N A$ and $(46, X Y)$ control DNA}

To test whether the differences in the numbers of $\mathrm{X}$ and $\mathrm{Y}$ chromosomes present in female and male genomes, and the 
Table 1. Evaluation of $18 \mathrm{CGH}$-metaphase spreads $(46, \mathrm{XY})$ subjected to CGH with $(47, \mathrm{XX},+21)$ genomic test DNA (TRITC-detection) and $(46, \mathrm{XY})$ control DNA (FITC-detection)

\begin{tabular}{|c|c|c|}
\hline & \multicolumn{2}{|c|}{$\begin{array}{l}\text { Mean of fluorescence ratio values }(F R) \\
\text { TRITC/FITC }\end{array}$} \\
\hline & $\begin{array}{l}\text { Measured } \\
\text { (lower and upper 95\% } \\
\text { confidence interval) }\end{array}$ & Expected \\
\hline $\mathrm{X}$ & $1.85(1.49 ; 2.43)$ & 2 \\
\hline Y & $0.19(0.12 ; 0.63)$ & $\cong 0$ \\
\hline 21 & $1.78(1.61 ; 2.0)$ & 1.5 \\
\hline All other autosomes & $0.99(0.82 ; 1.23)$ & 1.0 \\
\hline
\end{tabular}

difference between genomes with trisomy 21 and disomy 21, could be reliably detected by $\mathrm{CGH}$ experiments, digoxigenin-labeled genomic test DNA from a Down syndrome patient $(47, \mathrm{XX},+21)$ was mixed $(1: 1)$ with biotin-labeled genomic control DNA from a male individual $(46, X Y)$. After CISS hybridization to CGH-metaphase spreads $(46, X Y)$ and detection of the test and control DNA with TRITC and FITC, respectively, FRs for FITC/TRITC were measured in 18 metaphase spreads for chromosomes $21, \mathrm{X}, \mathrm{Y}$ and a control group consisting of all other autosomes. Chromosome identification was performed by DAPI banding (not shown). Figure 4 presents a typical FR image of a CGH-metaphase chromosome spread. The results are summarized in Table 1 and compared with the theoretically expected values. The $F R$ obtained for the chromosomes $\mathrm{X}, \mathrm{Y}$ and 21 were significantly $(P<0.001$; Student $t$-test $)$ different from the $F R$ obtained for all other autosomes. These results demonstrate that differences between test and control genomes regarding the copy numbers of the sex chromosomes and of chromosome 21, the smallest chromosome of the human complement, can be detected by $\mathrm{CGH}$.

\section{Detection of complete and partial chromosome gains and losses in tumor DNA samples by CGH}

For a rigorous test of the CGH approach, experiments were performed using two tumor DNA samples. The DNA samples were prepared from the cell line ACHN established from a papillary renal cell carcinoma and from peripheral blood cells of a patient with T-PLL. Cytogenetic analyses were performed in the laboratories of G. K. and H. D., whereas CGH analyses were carried out in the laboratories of T. C. and P. L. Information on the karyotypes of the two tumors was only shared between the laboratories after the full CGH analyses were completed and had yielded a fully independent proposition on chromosome gains and losses in the two tumor samples.

Chromosome imbalances detected by conventional banding and $C G H$ in the papillary renal carcinoma cell line ACHN

The karyotype obtained for cell line ACHN by GTG-banding analysis was: $53, \mathrm{X},-\mathrm{Y},+\operatorname{der}(1) \mathrm{t}(1 ; 10)(\mathrm{p} 13.1 ; \mathrm{q} 11.2),+2,+7$, $+7,+12,+12,+16,+17$ [10] (Fig. 5). CGH-metaphase spreads $(46, X Y)$ were hybridized with a $1: 1$ mixture of biotinylated ACHN tumor DNA (detected with FITC) and digoxigeninlabeled control DNA prepared from blood of a healthy male $(46, \mathrm{XY})$ (detected with TRITC). Figure 6 shows CCD images from a typical metaphase chromosome spread stained with TRITC (Fig. 6a), FITC (Fig 6b) and DAPI (Fig. 6c). A brief inspection of the chromosomes painted with the test DNA (Fig. 6b) shows that some of the autosomes are clearly more intensely painted than others. Such differences are not obvious for autosomes painted with control DNA (Fig. 6a).

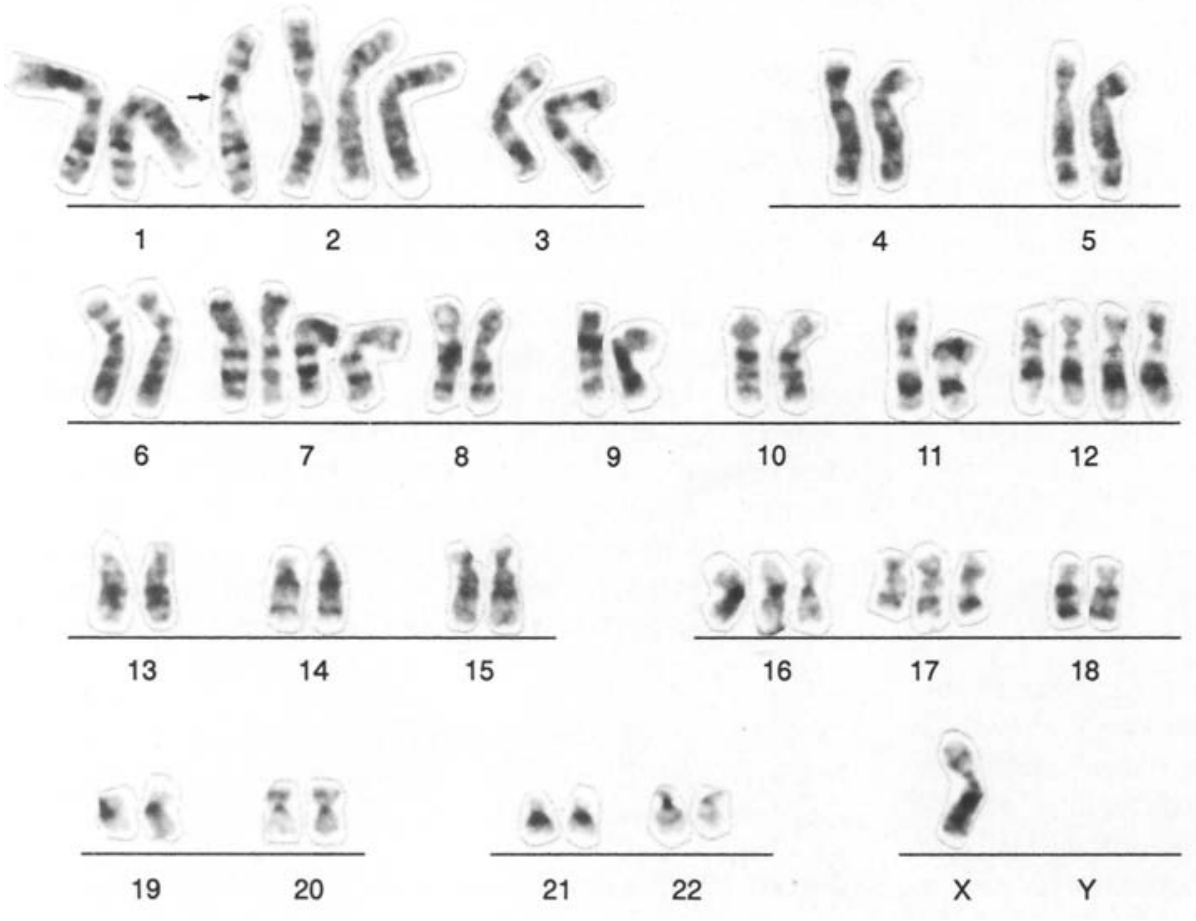

Fig. 5. G-banded karyotype of the cell line ACHN established from a papillary renal carcinoma. 53,X, $-\mathrm{Y},+\operatorname{der}(1) \mathbf{t}(1 ; 10)$ (p13.1;q11.2) $+2,+7,+7,+12,+12,+16,+17$. Arrow indicates the $\operatorname{der}(1) \mathrm{t}(1 ; 10)$ marker chromosome 

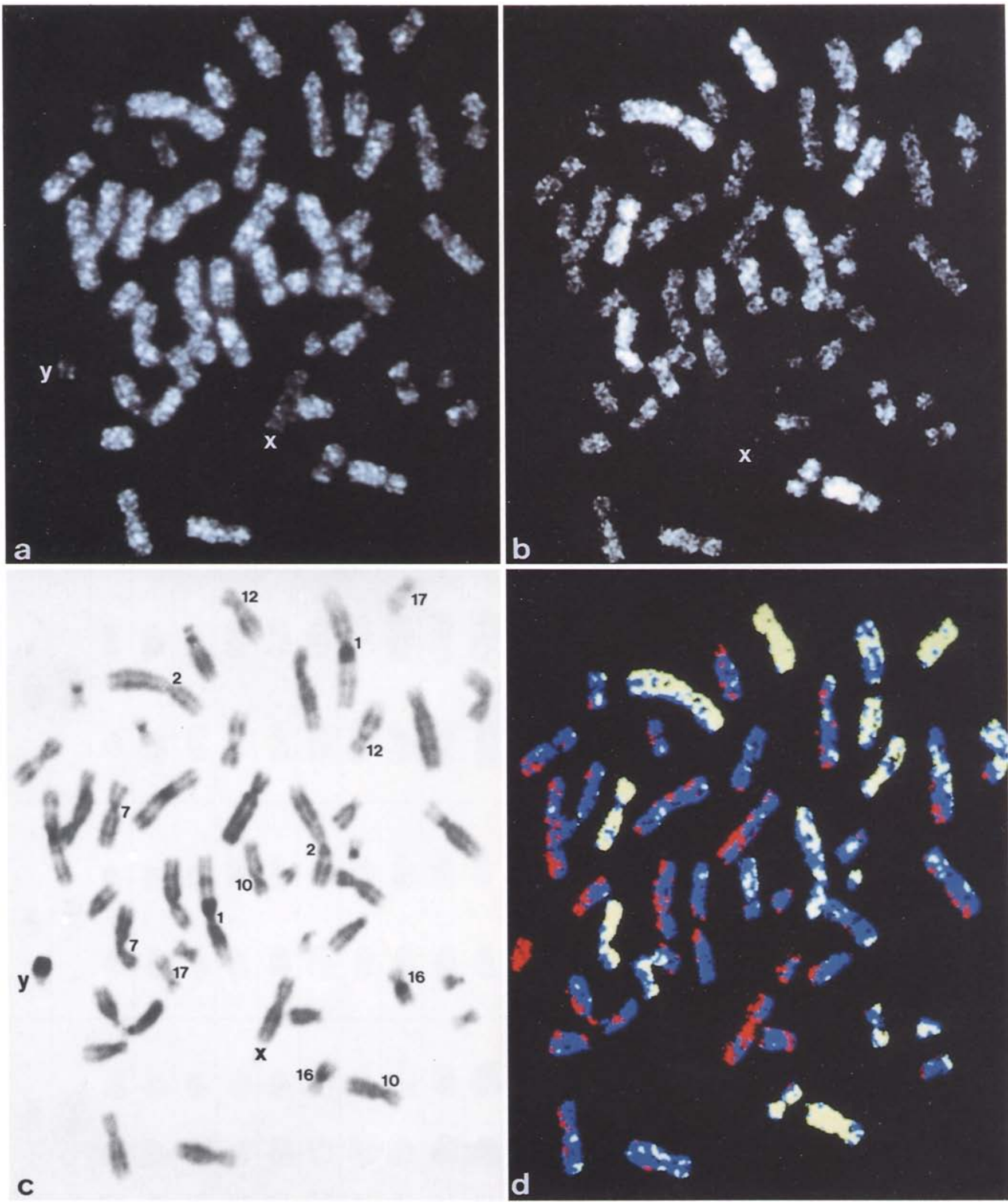

Fig. 6a-d. CGH-metaphase hybridized with renal carcinoma ACHNtest DNA detected with FITC, and (46,XY)-control DNA detected with TRITC. CCD images were acquired with filter blocks specific for a TRITC, b FITC, $\mathbf{c}$ DAPI. For better visualization of DAPI banding, the gray level image was inverted. Autosomes (nos. 1, 2, 7, 10, 12, 16, 17) for which complete or partial over-representation in the ACHN-test genome could be confirmed by both conventional chromosome banding analysis (Fig. 5) and evaluation of a series of CGH-metaphase spreads (see Figs. 7-11) are indicated in c. Notably, in both $\mathbf{a}$ and b, the X chro-

mosome is weakly painted. The euchromatic part of the $\mathrm{Y}$ chromosome is painted in $\mathbf{a}$, but not in $\mathbf{b}$. d FR image of the same metaphase representing a pixel by pixel display of FR values obtained with FITC/ TRITC. Blue suggests a balanced state of chromosome material; green suggests over-representation in the test genome; red under-representation. Whereas some chromosomes are homogeneously colored blue, green or red (e.g., Y), a predominant color is less obvious for others (compare Fig. 7) 


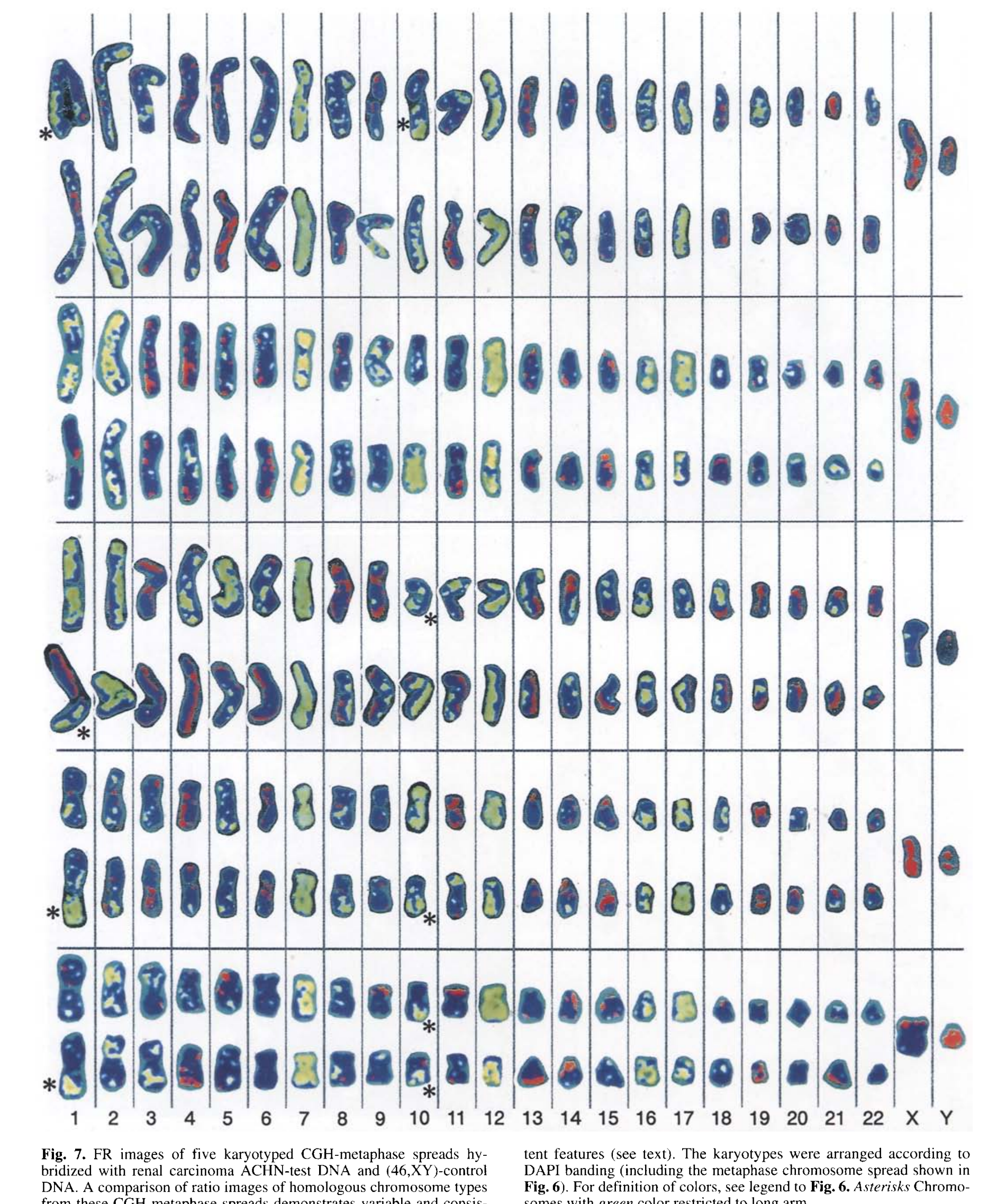



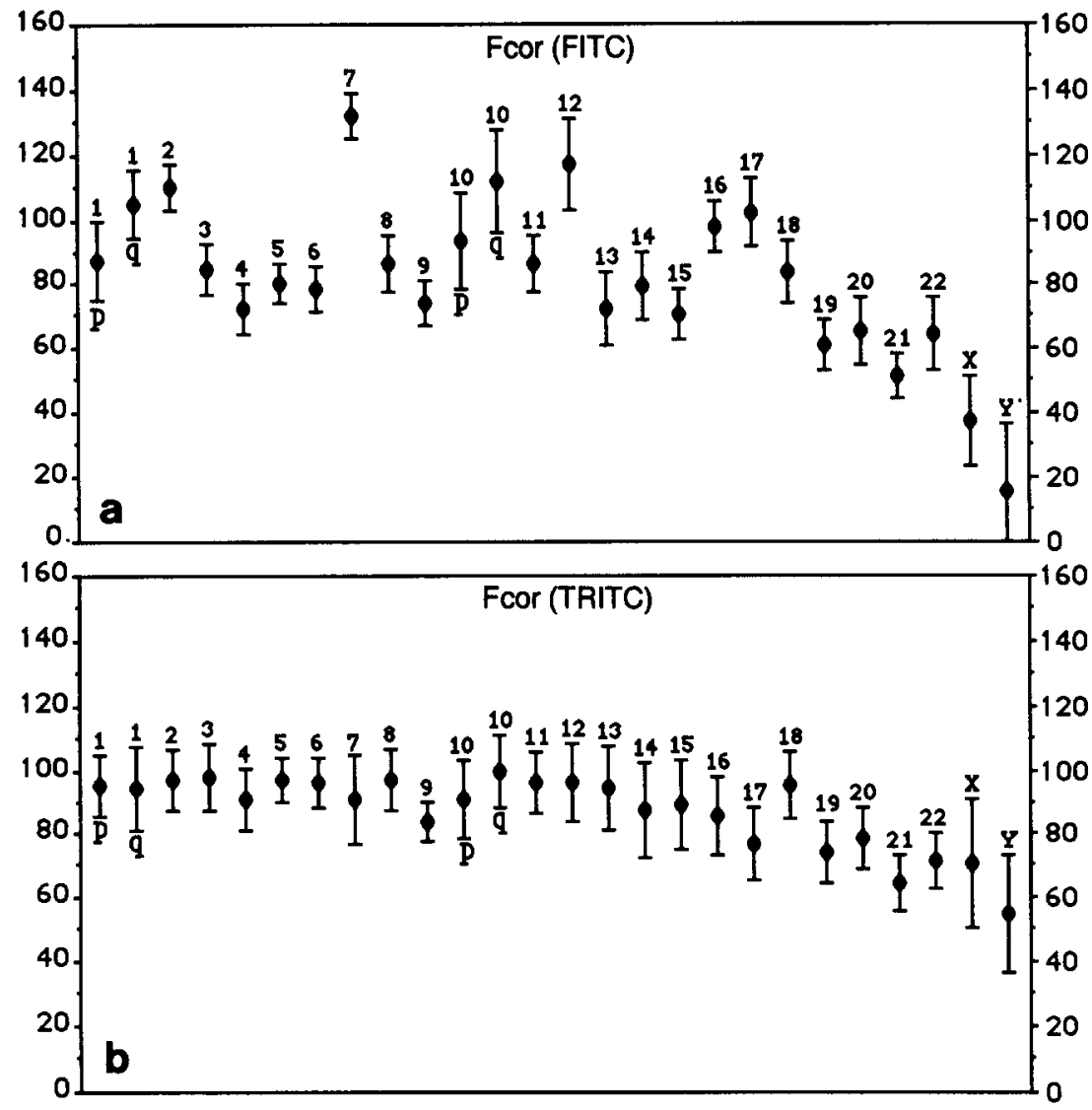

Fig. 8a, b. Means of FITC (a) and TRITC (b) fluorescence intensities [Fcor(FITC);Fcor(TRITC)] in $9 \mathrm{CGH}$-metaphase spreads hybridized with renal carcinoma ACHN-test DNA and $(46, \mathrm{XY})$-control DNA
For the $\mathrm{Y}$ chromosome, painting of the euchromatic part is demonstrated in Fig. 6a (test DNA), whereas no painting is seen in Fig. 6b (control DNA), indicating the absence of the $\mathrm{Y}$ chromosome from the tumor genome. In Fig. 6a, b, the X chromosome is apparently less intensely painted than most autosomes, suggesting the under-representation of the $\mathrm{X}$ chromosome in both genomes (see also below). A weaker painting of tandemly repetitive DNA sequences contained in the constitutive heterochromatic regions is noted in numerous chromosomes for both test and control DNA. This observation can be explained as an effect of signal suppression with Cot1 DNA (compare Fig. 13). A fluorescence ratio image of this metaphase is shown in Fig. 6d.

For a comparison, Fig. 7 shows karyotypes from $5 \mathrm{CGH}-$ metaphase spreads. Instead of the conventional banding pattern, the pixel by pixel image ratio is presented for each chromosome. A comparison of the image ratios for individual chromosomes demonstrates the profound variability of relative FITC and TRITC fluorescence intensities detectable not only between different chromosome types but even between some homologs. Numerous chromosomes are preferentially colored blue, indicating their balanced representation in the ACHN test genome. Other chromosomes, such as chromosomes $7,12,16$ and 17 are consistently colored green, indicating their over-representation. Chromosomes 1, 2, 10 are also preferentially, but less consistently, colored green, with several chromosomes 1 and 10 showing the green color restricted to the long arm (marked by asterisks). The $\mathrm{X}$ chromosomes are preferentially colored red, suggesting some under-representation.
In order to decide which chromosome or chromosome arm might be truly over-represented or underrepresented in the ACHN test genome, chromosomes from $9 \mathrm{CGH}$-metaphase spreads were evaluated in detail by two procedures. One evaluation procedure was based on $F R$ measured for each chromosome type and the definition of thresholds established from control $\mathrm{CGH}$ experiments. The second was based on the classification of chromosomes in the same series of metaphase spreads by visual comparison of FITC and TRITC images, as seen by CCD, into three categories (balanced, over-represented and under-represented chromosomes), followed by statistical analyses of the observed frequencies compared with expected frequencies in cases of random assignments.

Diagnosis of chromosomal imbalances in the ACHN test genome based on FR measurements. The means of the FITC and TRITC fluorescence intensities obtained for each chromosome type are shown in Fig. 8. As expected, the range of the FITC values (representing the tumor DNA) was much larger than the range of the TRITC values (representing the control DNA). The highest FITC value was found for chromosome 7, the lowest for the $Y$ chromosome. In contrast, the TRITC value for chromosome 7 was not significantly different from other C-group chromosomes, and the decrease of the TRITC value obtained for the $\mathrm{Y}$ chromosome was much less pronounced.

The FR calculated for each individual chromosome is presented in Fig. 9. Since FR images and visual inspection of CCD images had suggested a possible difference of FITC in- 


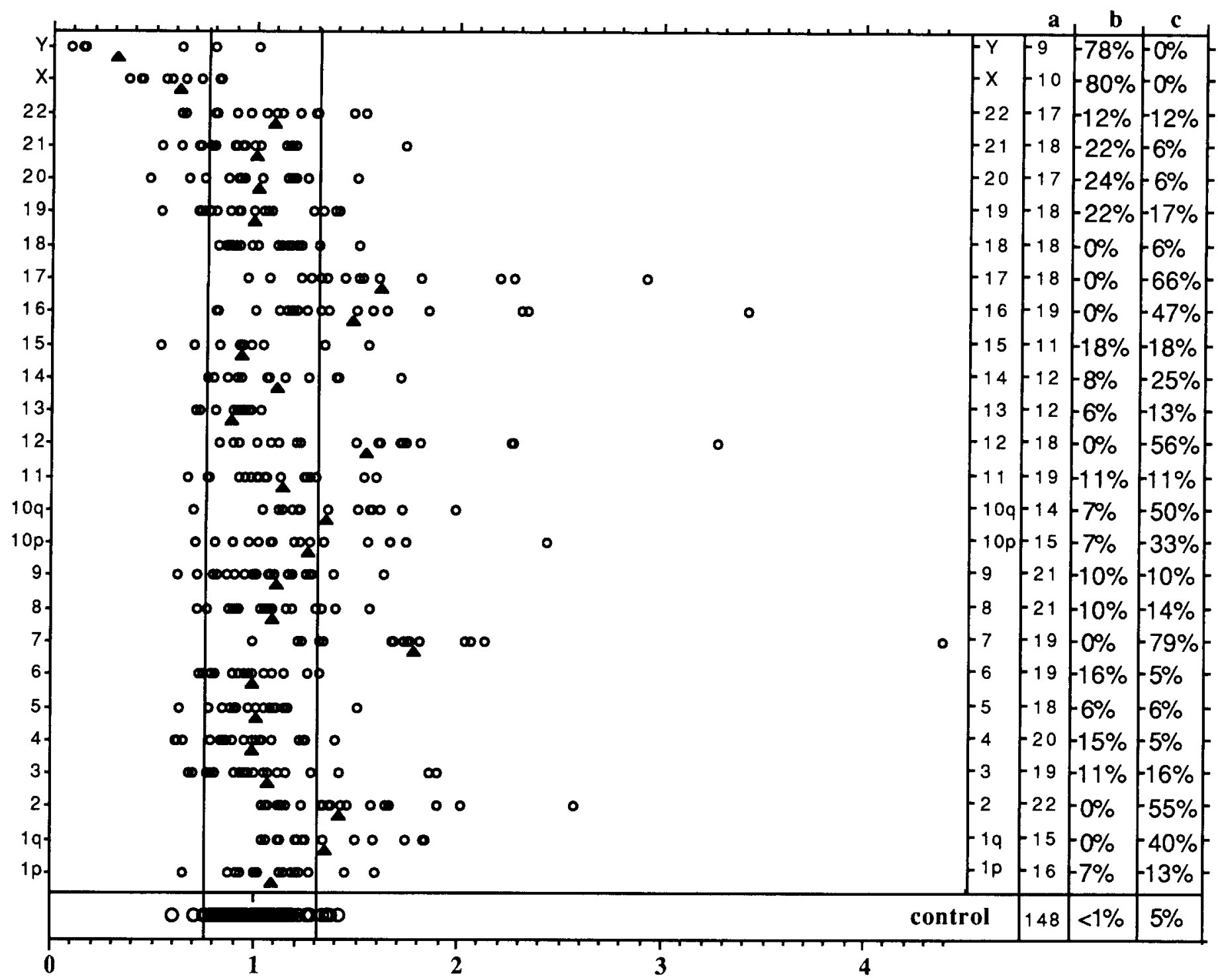

Fig. 9. FR values measured for chromosomes of nine $\mathrm{CGH}$-metaphase spreads hybridized with renal carcinoma ACHN-test DNA and (46,XY)-control DNA (upper part). Each point represents the FR of an individual chromosome; arrowheads represent the $F R$ for each chromosome type. For chromosomes 1 and 10, inspection of ratio images suggest a difference in the representation of the short and long arm (compare Fig. 7). Therefore, FR values were determined separately for both arms of these chromosomes. The bottom line presents FR values of chromosomes from three control CGH-metaphase spreads hybridized with differently labeled control genomic DNA $(46, \mathrm{XY})$ (see Methods).

tensities between $1 \mathrm{p}$ and $1 \mathrm{q}$, and between $10 \mathrm{p}$ and $10 \mathrm{q}$, the FRs were calculated separately for these chromosome arms. For an interpretation of these results, we defined a lower and upper threshold for $F R$. For this purpose, a control CISS hybridization experiment was carried out using a $1: 1$ mixture made from biotin-labeled and digoxigenin-labeled control genomic DNA $(46, X Y)$ detected with FITC and TRITC, respectively. In three metaphase spreads, FR values were determined for all chromosomes. (Fig. 9, bottom). $F R$ was calculated for this control chromosome population, and the limits of the $95 \%$ confidence interval were used as the lower and upper threshold (indicated as vertical lines in Fig. 9). Chromosome types for which $F R$ was beyond the threshold were
The two vertical lines indicate the lower and upper thresholds described in detail in the text (compare also Fig. 10). The columns on the right represent the following. (a) Number of chromosomes or chromosome arms used for FR measurements (for details, see legend to Fig. 3). Deviations from the expected numbers for each chromosome type arise because, in some cases, two masks were created for an individual chromosome (compare legend to Fig. 3), whereas in other cases, chromosomes were excluded because of chromosomal overlap. (b) Percentage of chromosomes with FR values above the upper threshold. (c) Percentage of chromosomes with FR values below the lower threshold considered to be under-represented or over-represented in the tumor. The $F R$ obtained for these chromosomes were compared with the theoretically expected FR values ( 2 for tetrasomy, 1.5 for trisomy, 1 for disomy, 0.5 for monosomy, 0 for nullosomy). The possible degree of over-representation or under-representation for a given chromosome type was assumed to be reflected by the nearest theoretical value (Fig. 10). Using these criteria, the following diagnosis was proposed for the representation of autosomes in the ACHN genome: tetrasomy 7 , trisomies $2,12,16$ and 17 , partial trisomies $1 \mathrm{q}$ and $10 \mathrm{q}$. This diagnosis is in complete agreement with the diagnosis independently obtained by conventional chromosome banding analysis with one exception: a trisomy 


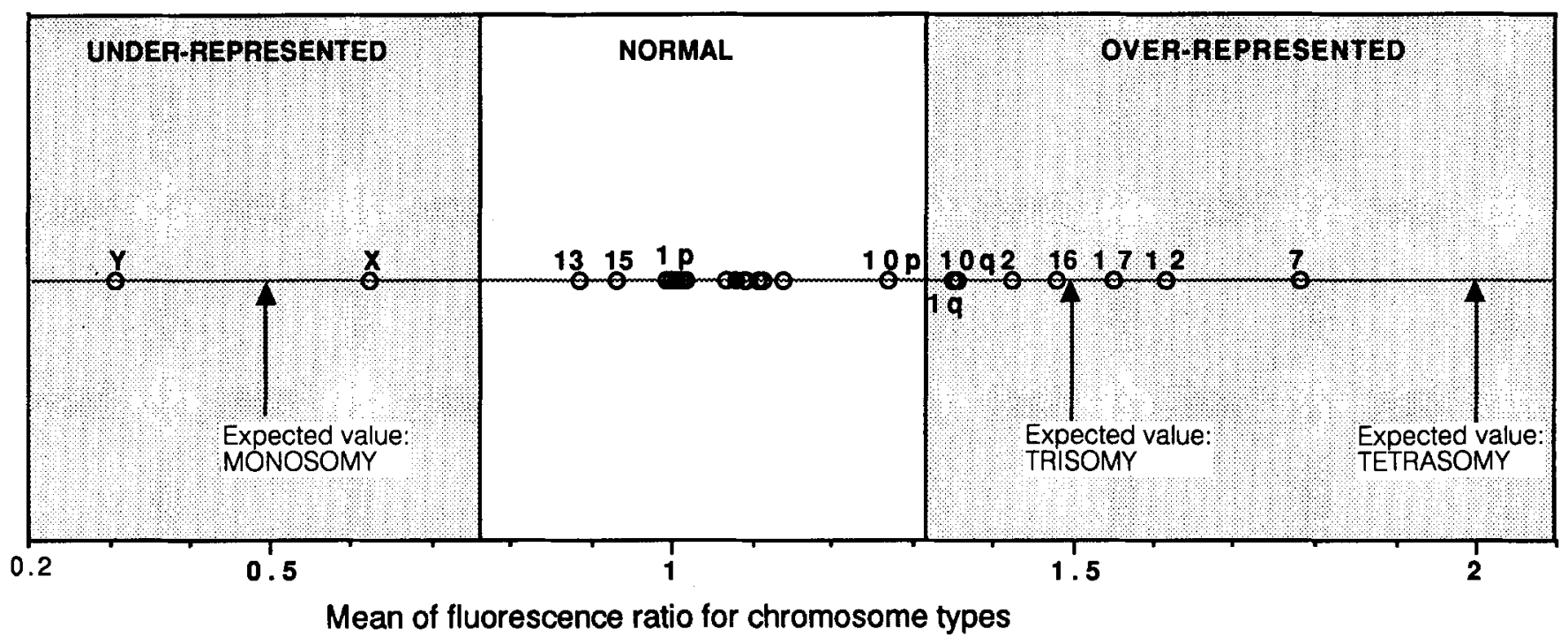

Fig. 10. Diagnosis of chromosomal imbalances in the ACHN test genome based on FR measurements. FR determined for each chromosome type in CGH-metaphase spreads hybridized with renal carcinoma ACHN test DNA and (46,XY)-control DNA (upper part) are presented together with the upper and lower thresholds determined from control CGH experiments (for details, see Fig. 9 and text). Chromosome types with $F R$ values within the threshold range were considered to be balanced in the test genome. Chromosome types 1q, 2, 7, 10q, 12, 16 and 17 showed $F R$ values above the upper threshold and were considered to be over-represented. The $F R$ value of the $Y$ chromosome indicates the absence of this chromosome in the tumor genome

was assumed for chromosome 12 by $\mathrm{CGH}$, whereas a tetrasomy was found by conventional analysis.

For the sex chromosomes, banding and $\mathrm{CGH}$ results indicated the lack of a Y chromosome in the ACHN cell line. However, since chromosome banding demonstrated the presence of a single $\mathrm{X}$ in both the control and the test genome, the $F R$ value obtained for the $\mathrm{X}$ chromosome provided a puzzle. Although an $F R$ value for the $\mathrm{X}$-chromosome close to 1 was expected, the value measured for the $\mathrm{X}$ chromosome was clearly below the lower threshold. Three reasons may be considered: (1) an artefact of the CGH approach. (2) loss of the $\mathrm{X}$ chromosome in a major subpopulation of the ACHN cell line, not detected by banding analysis, and (3) a relatively more intense painting of the $\mathrm{X}$ chromosome by homolog sequences of the $\mathrm{Y}$ chromosome contained in the control genome $(46, X Y)$, but not in the ACHN genome. Although recent findings indicate that homolog sequences are not restricted to the pseudoautosomal region (Koenig et al. 1985; Page et al. 1987), there is no evidence that such homologies would exist to an extent sufficient to explain the deviation in the measured FR. Indeed, chromosome painting using DNA from sorted $\mathrm{Y}$ chromosomes as a probe yields hybridization signals on Xp22.3 and Xq13, but does not result in a uniform $X$ chromosome painting (Jauch et al. 1990).

Diagnosis of chromosomal imbalances in the ACHN test genome based on visual inspection of CGH images. Visual examination of CCD images from CGH-metaphase spreads by several investigators gave the impression that certain

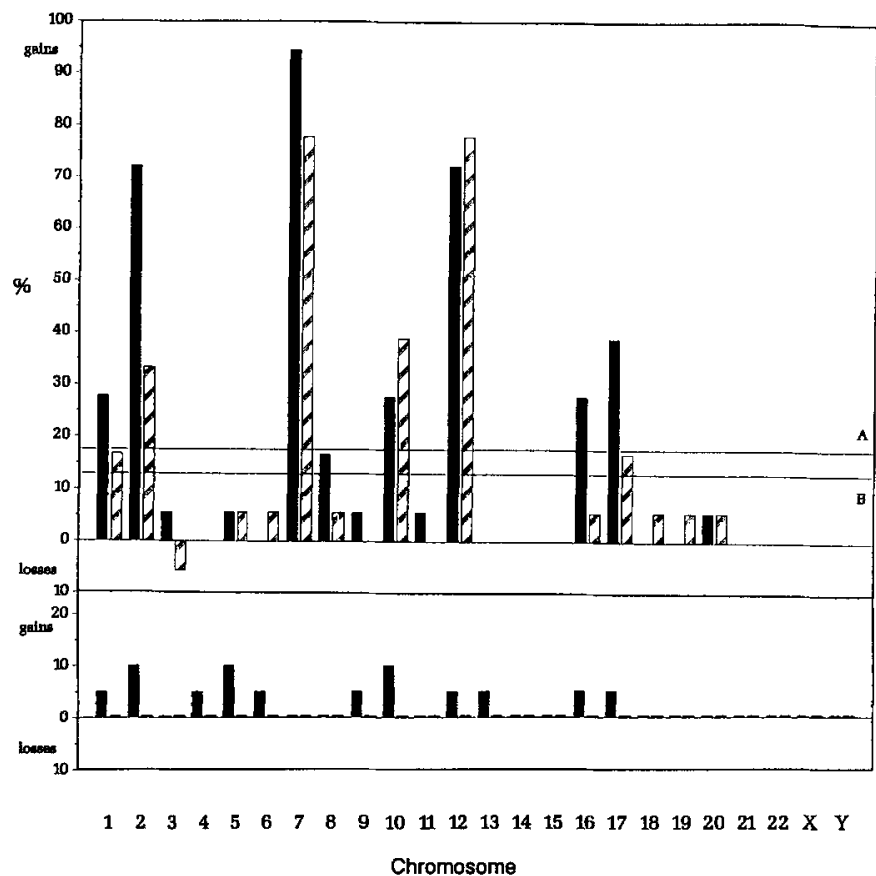

Fig. 11. Top Diagnosis of chromosomal imbalances in the ACHN test genome based on visual inspection of $\mathrm{CCD}$ images of nine $\mathrm{CGH}$ metaphase spreads. Abscissa Chromosome types evaluated in CGHmetaphase spreads. Ordinate Frequencies with which each chromosome type was marked as being suspicious for over-representation (gains) or under-representation (losses) of a given chromosome by two independent observers A (solid bars) and B (hatched bars). The observers considered only whole chromosomes as objects and did not try to identify chromosome areas suspicious for partial gains and losses (e.g., 1q, 10q; compare Fig. 10). The two horizontal lines $A$ and $B$ indicate the frequency of markings expected for each of the two observers in cases of a random assignment. Using these frequencies as thresholds, the data obtained by observer $A$ indicate the over-representation of chromosomes $1,2,7,10,12,16$ and 17. Data from observer B indicate over-representation of the same chromosomes with the exception of chromosome 16. None of the two observers detected the absence of the $\mathrm{Y}$ chromosome in the test genome. Bottom Visual inspection of CCD-images from ten control CGH-metaphase spreads. Control CGH-metaphase spreads were evaluated as above. Observer A marked 14 chromosomes of different sizes as being suspicious for a gain in the test genome, whereas no chromosomes were marked by observer $B$ 

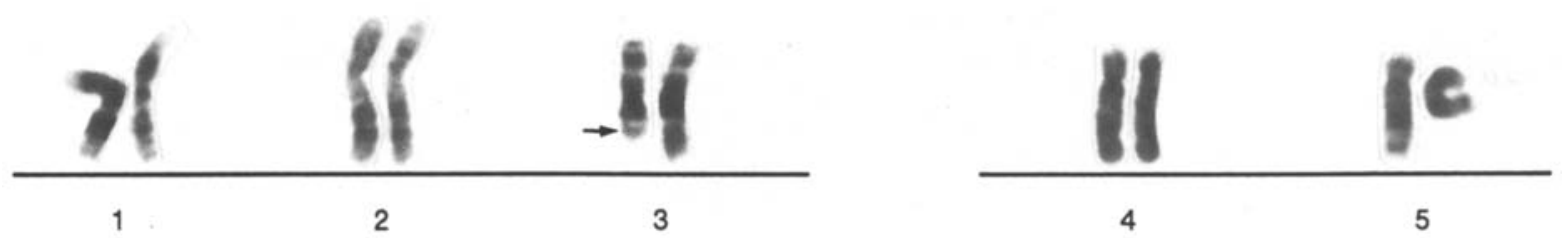
3
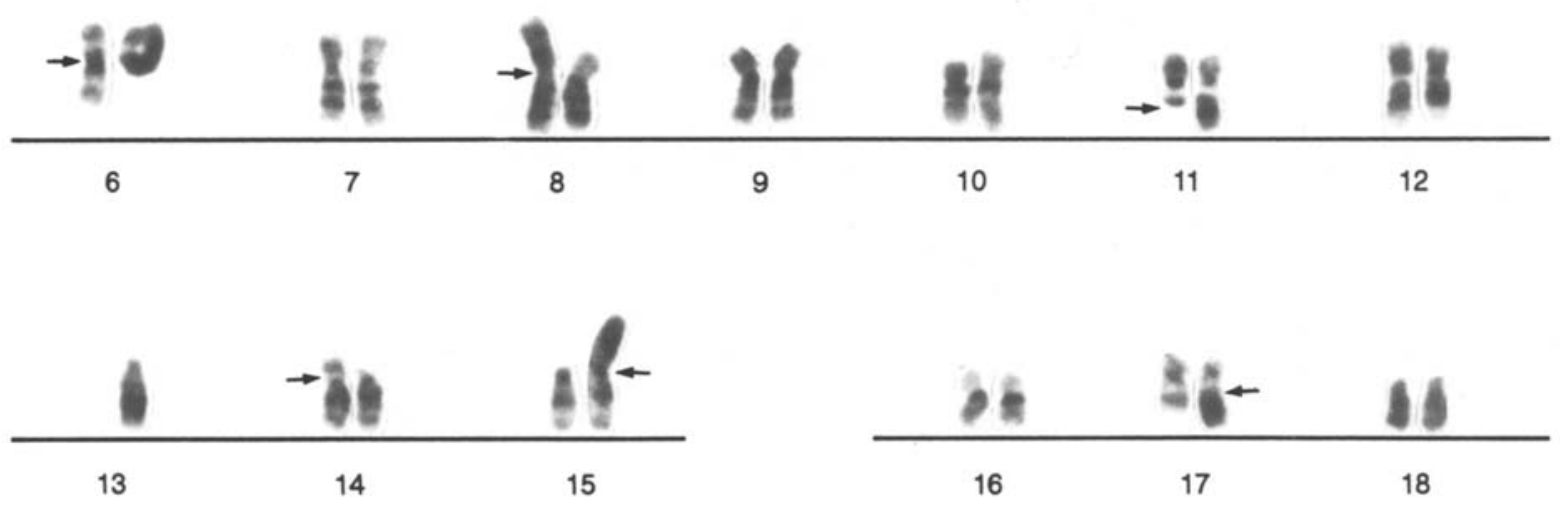

16

17

18
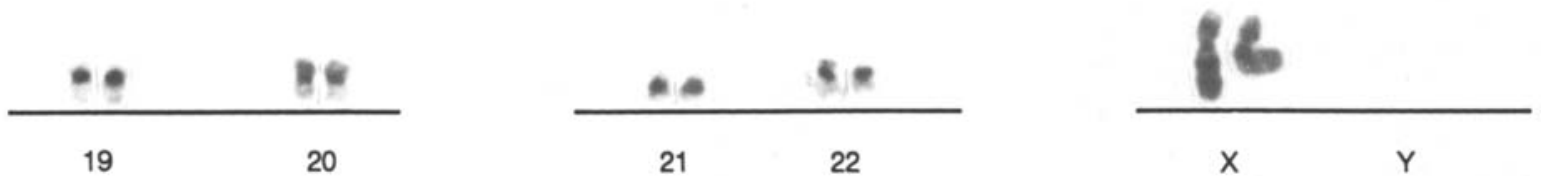

Fig. 12. G-banded karyotype from clone 2 of the patient with T-PLL. $45, X X, t(3 ; 17)(q 24 ; q 21), i(6 p), i(8 q), \operatorname{del}(11)(q 21), \operatorname{der}(13 ; 15)(q 10 ; q 10)$, add (14)(pl1). Marker chromosomes are indicated by arrows. The $t(3 ; 17)$ was not observed in the second clone 2 metaphase spread and is therefore not considered as a clonal aberration by tumor cytogenetic conventions

chromosomes considered suspicious for gains or losses in the ACHN genome might be identified by visual inspection of CCD images without the necessity of FR measurements. Since such an evaluation would be of interest for laboratories that are equipped with a CCD camera but that have no expertise in quantitative image analysis, we tested its feasibility. Two observers A and B were asked independently to perform a visual side-by-side comparison of TRITC and FITC images obtained by $\mathrm{CCD}$ from the nine $\mathrm{CGH}$-metaphase spreads previously evaluated by FR measurements (see above), and from ten control $\mathrm{CGH}$-metaphase spreads hybridized with a 1:1 mixture of differently labeled normal genomic DNA $(46, X Y)$. Pairs of FITC and TRITC images from the control and tumor $\mathrm{CGH}$ experiments were presented in random order. Observers were asked to compare each pair of FITC and TRITC images and mark chromosomes that they judged to present a higher or lower fluorescence intensity after painting with the test DNA (FITC) relative to the majority of the chromosomes (for further details see Methods). The results are summarized in Fig. 11 and compared with the threshold frequencies expected if the chromosomes chosen by each observer were marked at random. Using these criteria, the following chromosomes were considered as candidates for over-representation in the test genome: $1,2,7,10,12,16$ and 17 (data obtained by observer A) and 1, 2, 7, 10, 12 and 17 (data obtained by observer B). On questioning, both observers had recognized the weaker painting of the $X$ chromosome in CCD images from test and control DNA, but following the instructions given to them (see Methods), they had not marked this chromosome. The loss of the $\mathrm{Y}$ chromosome in the ACHN genome was missed by both observers for two reasons. First, this chromosome was not painted by the test DNA and, secondly, in absence of a DAPI image, painting of the small euchromatic part of the $Y$ chromosome by the control DNA was overlooked or considered to be a background artefact.

In the ten control CGH-metaphase spreads, observer B never marked a chromosome, whereas observer A marked 14 chromosomes as being suspicious for over-representation in the test genome. Observer A also marked more chromosomes (73) than observer B (54) in the nine test CGH-metaphase spreads. These differences suggest that observer A considered as suspicious smaller differences of painting intensities than observer B. Interestingly, the data obtained by observer A led to the identification of all chromosome over-represented in the ACHN test genome, whereas the trisomy 16 was missed by the evaluation of observer $B$.

\section{Chromosome imbalances detected by CGH in T-PLL cells}

To investigate further the potential of $\mathrm{CGH}$, we also performed a study of primary tumor cells represented in high proportions in the blood of a female patient suffering from $T$ - 


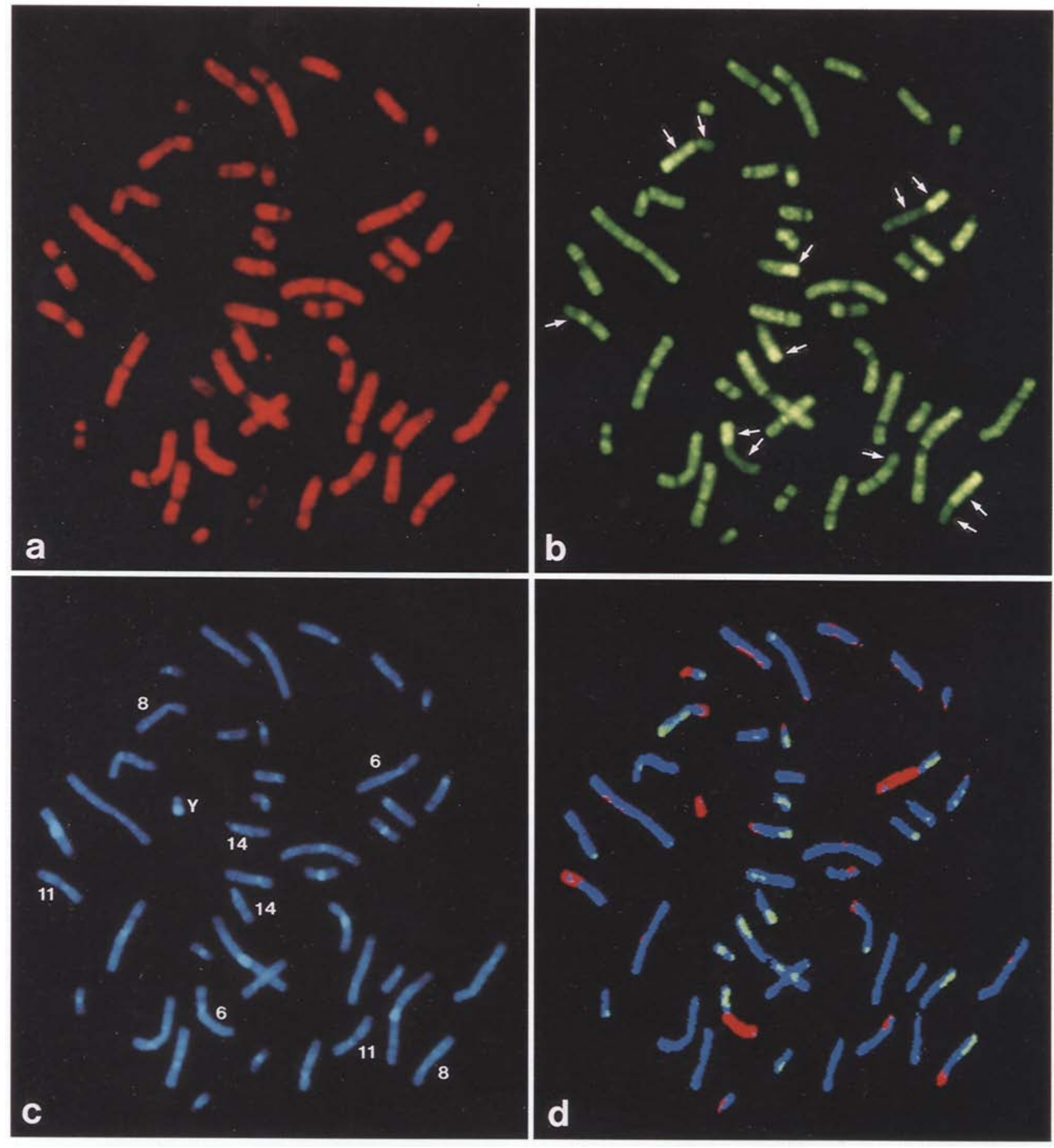

Fig. 13a-d. CGH-metaphase hybridized with T-PLL-test DNA detected with FITC and $(46, \mathrm{XX})$-control DNA detected with TRITC. CCD-images acquired for a TRITC, b FITC, $\mathbf{c}$ DAPI. Visual inspection of $b$ reveals weak staining of $6 \mathrm{q}, 8 \mathrm{p}, 11 \mathrm{q} 21-\mathrm{qter}$ and $\mathrm{Y}$. Strong staining is found on $6 \mathrm{p}, 8 \mathrm{q}$ and $14 \mathrm{q} 24$-qter (arrows). An FR image of this metaphase is shown in $\mathbf{d}$ (for color code see legend to Fig. 6). Only one copy of chromosomes 2,10 and 17 is present in this $\mathrm{CGH}$-metaphase spread. Their blue color indicates the presence of two copies in the test genome. As expected for test and control DNA from female individuals, a very weak FITC and TRITC fluorescence was observed over the $Y$ chromosome. With such weak staining, FR measurements become unreliable. The red of the $\mathrm{Y}$ chromosome seen in $\mathbf{d}$ is therefore considered to be an artefact
PLL. The results obtained by CGH were compared with the results of conventional karyotype analysis performed on short term cultured cells. As in the case of the papillary renal cell carcinoma described above, information on the clinical diagnosis and the karyotype was only revealed after a fully independent diagnosis was obtained by $\mathrm{CGH}$.

Giemsa-Wright banding of the chromosomes revealed two clonal aberrations. Clone $1: 45, X X, \operatorname{dic}(6 ; 15)(q 11 ; p 11), i(8 q)$, $\operatorname{del}(11)(\mathrm{q} 21),-13,+\operatorname{mar}[15]$. This karyotype presents a monosomy 13 , partial monosomies $6 \mathrm{q}, 8 \mathrm{p}, 11 \mathrm{q} 21-\mathrm{qter}$, and a partial trisomy $8 \mathrm{q}$. The origin of the additional small marker 
chromosome could not be identified by banding. Clone 2 : 45, XX,i(6p), i(8q), del(11)(q21), der(13;15)(q10;q10), add(14) (p11) [2]. In addition to the partial monosomies $6 q, 8 p$ and $11 \mathrm{q} 21$-qter, and the partial trisomy $8 \mathrm{q}$ already observed in clone 1 , the karyotype of clone 2 shows a partial trisomy $6 \mathrm{p}$ and a derivative chromosome 14 with additional material on the short arm (Fig. 12). Moreover, clone 2 contains a Robertsonian translocation $t(13 ; 15)$. A karyotype of one of the two metaphase spreads observed from clone 2 is presented in Fig. 12. In routine clinical diagnostics, 10 out of 20 analyzed metaphase spreads originally evaluated from this patient showed the clone 1 karyotype. One metaphase spread showed the clone 2 karyotype, but initially was not considered as a separate clone following the conventions of tumor cytogenetics (but see below).

CGH was carried out using a 1:1 mixture of biotinylated genomic test DNA prepared from peripheral blood of the patient and digoxigenin-labeled control DNA $(46, X X)$. Detection of the test DNA was performed with FITC (or Texas red), whereas detection of the control DNA was achieved with TRITC (or FITC). Independently of the combination of fluorochromes used for the detection, a number of chromosomal segments suspicious for gains or losses in the test genome was readily observed by conventional fluorescence microscopy. CCD images of CGH-metaphase spreads were acquired for TRITC (control DNA) (Fig. 13a), FITC (test DNA) (Fig. 13b) and DAPI (Fig. 13c) staining. Figure 13d presents the fluorescence image ratio of the metaphase spread presented in Fig. 13.

Two observers (C and D) independently evaluated FITC and TRITC images obtained by $\mathrm{CCD}$ from $23 \mathrm{CGH}-$ metaphase spreads by visual inspection (see Methods). Observers in this experiments were requested to mark not only whole chromosomes, but also chromosome arms suspicious for over-representation or under-representation. The results are shown in Fig. 14 and compared with the threshold frequencies expected for random assignments (see Methods). Using these criteria, the data from both observers revealed over-representation of $6 \mathrm{p}, 8 \mathrm{q}$ and $14 \mathrm{q}$, and under-representation of $6 \mathrm{q}, 8 \mathrm{p}$, distal $11 \mathrm{q}$ and $16 \mathrm{q}$. In addition, over-representation of $16 \mathrm{p}$ and 22 was found by observer $\mathrm{D}$ but not by observer $\mathrm{C}$. The chromosomal breakpoints on $11 \mathrm{q}$ and 14 were further characterized by comparison of CCD images from chromosomes 11 and 14 in CGH-metaphase spreads painted with the tumor DNA and banded with DAPI (Fig. 15). This analysis identified the deleted region of chromosome 11 as del(11)(q14or21-qter). The over-represented region of chromosome 14 was defined as $14 \mathrm{q} 24$-qter.

Over-representation of $8 \mathrm{q}$ and under-representation of $6 \mathrm{q}$, $8 \mathrm{p}$ and the distal part of $11 \mathrm{q}$ were in agreement with the results of banding analyses. In contrast, although chromosome arms $6 p$ and $14 q$ were marked with high frequencies by both observers as candidates for over-representation, these findings did not fit with the karyotype obtained for clone 1, the only clone originally detected. This prompted a more extended evaluation of the banded metaphase spreads from the patient, resulting in the identification of clone 2. In total, 40 metaphase spreads were analyzed, 15 showed the clone 1 karyotype, 2 the clone 2 karyotype and 23 a normal karyotype. The results of CGH analysis are in agreement with the karyotype of clone 2. It is known that the proportions of var- ious clones contained in the blood of patients and detected in short term cell cultures may differ largely. Thus, the possibility has to be considered that clone 2 was predominant in the blood from which the test DNA was prepared, although it was detected only in a low proportion of the cells analyzed by conventional chromosome analysis after short term culture. In this case, the results for chromosomes 6, 13 and 14 obtained by banding analysis of clone 2 and $\mathrm{CGH}$ analysis could be fully reconciled.

The CGH analysis of chromosome 14 would fit with the interpretation that the additional material detected on the short arm of the $14 p+$ chromosome in the two clone 2 metaphase spreads represents the region 14q24-qter (Fig. 15 ). Recurrent aberrations of chromosome 14, including inversions and translocations with a breakpoint in $14 \mathrm{q} 32.1$, have been described in T-cell lymphocytic leukemia (Matutes et al. 1991).

Painting of chromosome 13 in tumor metaphase spreads (not shown) demonstrated the presence of chromosome 13 material in the unidentified marker chromosome found in clone 1 in addition to the normal chromosome 13. Thus, chromosome 13 was balanced in clone 2 and at least partially balanced in clone 1 .

Three discrepancies between the results of CGH and Gbanding analyses still remain. Data from both observers indicate an under-representation of $16 \mathrm{q}$. In addition, the findings of observer $\mathrm{D}$, but not of observer $\mathrm{C}$, indicate the over-representation of chromosome arms 16p and chromosome 22. Since test genomic DNA was only available from the blood of the patient, but not from cultured tumor cells, we could not test whether a CGH analysis performed with the latter test DNA would be wholly compatible with the results of chromosome banding performed after short term culture. In addition, it is not yet clear whether polymorphisms of normal genomic DNAs obtained from other sources than the normal somatic cells of the patient may influence the results of a $\mathrm{CGH}$ analysis.

\section{Discussion}

$\mathrm{CGH}$ of test and control genomes was performed on normal metaphase chromosome spreads $(46, \mathrm{XX}$ or $46, \mathrm{XY})$ (CGHmetaphase spreads). Genetically imbalanced chromosomes in several test genomes could be rapidly detected, and chromosome segments could be mapped to their normal chromosome counterparts in CGH-metaphase spreads. Genomic test

Fig. 14. Diagnosis of chromosomal imbalances in the T-PLL test genome based on visual inspection of CCD images of $23 \mathrm{CGH}$ metaphase spreads. Abscissa Chromosome types evaluated in the CGHmetaphase spreads. Chromosome arms are listed separately in cases where observers detected significant differences in painting intensities over the two arms hybridized with test DNA. Ordinate Frequencies with which chromosome types were marked as suspicious for over-representation (gains) or under-representation (losses) by two independent observers C (solid bars) and D (hatched bars). For further explanation of thresholds, see legend to Fig. 11. The data obtained by observer $\mathrm{C}$ indicate the over-representation of $6 \mathrm{p}, 8 \mathrm{q}$ and 14 , and under-representation of $6 \mathrm{q}, 8 \mathrm{p}, \mathrm{l1q}$ and $16 \mathrm{q}$. The data obtained by observer D are consistent with these findings. In addition over-representation of $16 p$ and 22 was found 


$$
\|_{i}
$$


DNAs were prepared from blood of a patient with trisomy 21 , from a renal papillary carcinoma cell line, and from blood of a patient with T-PLL. In the following discussion, we will consider: (1) major advantages and limitations of CGHanalyses; (2) possible pitfalls and improvements; (3) an integrated approach based on the combination of chromosome banding, CGH and FISH with chromosome-specific DNA probes; (4) perspectives for genome analysis of normal and pathological cell populations.

\section{Major advantages and limitations of $\mathrm{CGH}$}

CGH provides a new global approach for searching clinical and tumor specimens for genetic imbalances in a single CISS hybridization experiment. It avoids possible pitfalls of cell culture, can be performed in cases where genomic DNA from the suspected cells is the only material available for analysis, and is less time consuming than other molecular genetic approaches presently used to search a genome for genetic imbalances (see Introduction).

Several limitations of CGH compared with conventional banding analyses also need to be emphasized. Balanced chromosome rearrangements cannot be detected. CGH does not provide any information regarding the way in which chromosome segments involved in gains and losses are arranged in marker chromosomes of the test genome. Finally, chromosomal imbalances can only be detected if they are present in most cells of the specimen. Thus, CGH cannot be applied to studying the clonal heterogeneity of the test specimens. On the other hand, in cases of tumors with instable karyotypes, the fact that random gains and losses of chromosome material affecting only a few cells cannot be ascertained should help tremendously in distinguishing chromosomal imbalances occurring at random in only a few tumor cells from changes present in the majority of cells of a given tumor. $\mathrm{CGH}$ analyses performed with tumor DNAs prepared from a series of individual tumors representing a distinct tumor type should lead to the identification of those chromosomal imbalancies that are consistently involved, and should thus help to identify candidate chromosome segments for genes of major biological importance for the tumor type in question.

\section{Pitfalls and possible improvements of $\mathrm{CGH}$ and image analysis}

Possible pitfalls need to be carefully studied and the procedures validated with a large number of clinical cases before $\mathrm{CGH}$ analyses can be recommended for routine clinical purposes. The optimal use of the techniques described in this paper requires familiarity with both cytogenetics and image analysis. Since only a minority of readers may be equally knowledgeable in both fields, the following discussion has been written to point out some particularly important technical aspects for the non-specialist in either field. The diagnosis of chromosomal imbalances in test genomes requires a statistical approach based on the analysis of a number of $\mathrm{CGH}^{-}$ metaphase spreads, since the range of individual FR values obtained for chromosome types present in normal and abnormal numbers in the test genome show considerable overlap. Technical improvements at various steps of the procedure can be implemented with the goal of reducing the extent of

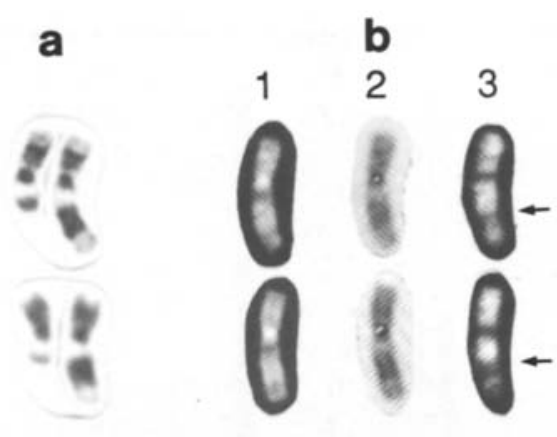

Chromosome 11

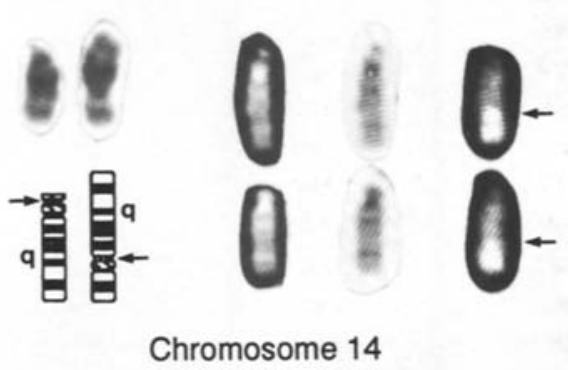

Fig. 15. Comparison of breakpoints of chromosomes 11 and 14 in TPLL cells identified by conventional G-banding analysis and $\mathrm{CGH}$ analysis. Column a G-banded normal and derivative chromosomes 11 (clone 1) and 14 (clone 2) from metaphase spreads of the patient with $\mathrm{T}$ PLL. Columns b / 3 Chromosomes 11 (upper part) and 14 (lower part) from a CGH-metaphase spread $(46, X X)$ hybridized with T-PLL test DNA and $(46, X X)$-control DNA. Column I CCD image of DAPIbanded chromosomes; column 2 the same $\mathrm{CCD}$ image with inverse representation of the gray values; column $3 \mathrm{CCD}$ image of FITC labeled test DNA. Arrows indicate the site of an rapid change in fluorescence intensities along chromosomes 11 and chromosome 14. For chromosome 11, banding and $\mathrm{CGH}$ analyses consistently indicate a deletion $\operatorname{del}(11)(\mathrm{q} 14$ or21-qter) with the breakpoint either in distal q 14 or at the border of the bands q14 and q21. For chromosome 14, the ideogram indicates the putative origin of additonal material: on the left, a normal chromosome 14 is presented, on the right, the $14 p+$ is turned around to indicate better the suggested correspondence of the additional material in this derivative chromosome with $14 \mathrm{q} 24-$ qter of the normal counterpart (arrows point to the centromeres). This assumption is in concordance with the over-representation of $14 \mathrm{q} 24$-qter detected by the $\mathrm{CGH}$ experiment

this overlap and of increasing the sensitivity of CGH analyses for the detection of partial gains and losses of chromosomes.

Quality tests of metaphase chromosome spreads used in CGH experiments. The quality of CGH strongly depends on the quality of the chromosome preparations. To minimize inter-experimental variability in CGH experiments, we recommend that large stocks of slides are prepared with high quality metaphase spreads (for optimum storage conditions, see Lichter and Cremer 1992). Each stock should be carefully tested for its suitability in $\mathrm{CGH}$ experiments. Control CGHexperiments with 1:1 mixtures of differently labeled normal genomic DNA should yield a uniform intense painting of all chromosomes (except for constitutive heterochromatin where reduced painting often occurs in CISS hybridization experiments, see Results). Whereas metaphase chromosome 
spreads with shorter and correspondingly thicker chromosomes facilitate the creation of segmentation masks used for fluorescence measurements, longer chromosomes are preferable for the identification of breakpoints involved in partial chromosomal gains and losses.

Optimization of CISS hybridization in CGH experiments. Careful adjustment of parameters, such as the size range of labeled DNA sequences, RNAse and protein digestion pretreatment steps, can all help to minimize background problems (for details, see Lichter and Cremer 1992). Cross-hybridization of interspersed repetitive DNA sequences contained in the labeled test and control DNAs diminishes the differences that can be expected between the FRs obtained for monosomic, disomic and trisomic chromosome segments in CGH experiments. For this reason, we have added extensive amounts of unlabeled Cotl fraction of human DNA to the hybridization mixture. Prehybridization of the chromosome spreads with the Cotl fraction also provides an effective reduction in the effects of cross-hybridization. We expect that minor variations in the proportions of test and control genomic DNA contained in the hybridization mixture should have little, if any, effect on the relative differences expected between the FRs measured for monosomic, disomic, trisomic segments, etc., provided that the relative error of quantitative fluorescence measurements does not increase because of weak hybridization signals of test and/or control DNA. Detectable differences in fluorescence intensities between balanced and unbalanced chromosome types of test genomes have also been noted in experiments where only test DNA was included in the hybridization mixture (see Joos et al. 1992). However, in the absence of control DNA, one should expect that the discrimination between chromosome types present in normal and abnormal numbers in the test genome requires optimally chosen hybridization times. Assuming (1) that the maximum hybridization efficiency possible for each chromosome type is not limited by the rapid renaturation of target DNAs, and (2) that an excess of labeled chromosome-specific sequences for all chromosomes is available in the hybridization mixture, extended hybridization times should lead to the complete coverage of the target sequences available on all chromosomes in CGH-test metaphase spreads with test DNA sequences, independent of whether these sequences are represented in a balanced or unbalanced state in the test genome. In contrast, the presence of differently labeled control DNA in addition to the test DNA ensures that an FR specific for the balanced or unbalanced state of each chromosome type in the test genome will build up during the complete time-course of the CISS hybridization. In the latter case, we would expect that saturation of the chromosomes with differently labeled sequences from both genomic DNAs should greatly facilitate the measurement of accurate ratio values. Instead of DNA probes labeled with different haptens, recent experiments have shown that it is also possible to use DNA probes directly conjugated to fluorochromes in various proportions for FR measurements (own unpublished data).

Optimization of image acquisition. The quality of FR measurements depends on the uniformity with which CGHmetaphase chromosome spreads can be illuminated (e.g., by a mercury lamp) and the accuracy with which overlays of CCD images obtained with different filter sets can be produced (Aikens et al. 1989). A single multi-bandpass dichroic mirror (Bright et al. 1989) optimally adapted for the fluorochromes used for FR measurements can help to avoid image shifts resulting from mirror changes when gray level $\mathrm{CCD}$ images are successively acquired for each fluorochrome. Sets of multi-bandpass filters (Hiraoka et al. 1991; Kaplan et al. 1992) in combination with a color CCD camera useful for the simultaneous quantitative assessment of several fluorochromes are presently being developed (de Lange et al. 1992) and should also help to avoid any pixel shifts. The simultaneous recording of the emission light from two or three fluorochromes using a laser scanning microscope equipped with two or three photomultipliers (Humbert et al. 1992) may provide another possibility for performing ratio fluorescence measurements on a pixel by pixel basis. At the same time, contributions of out-of-focus fluorescence can be minimized by working in the confocal mode (Robert-Nicoud et al. 1989).

Optimization of evaluation procedures. Two evaluation procedures, based on FR measurements and visual inspection of CCD images, respectively, have been used in the present CGH experiments.

The normal range of FR values, indicating a balanced state of a chromosome type in the test genome, was deduced from a control CGH experiment performed with a 1:1 mixture of differently labeled control genomic DNA. Alternatively, internal standardization seems possible, considering the fact that, in all chromosome syndromes and in most tumors, genetic imbalances affect only a minority of the chromosome types. Accordingly, it seems reasonable to deduce the normal range of FR values empirically from a number of chromosome types showing $F R$ values close to the value expected for balanced chromosomes.

Although an evaluation of CGH-metaphase spreads based on FR measurements provides the most objective and reliable method, evaluation by visual inspection of CCD images could help to introduce the $\mathrm{CGH}$ approach in laboratories that are not equipped at present to perform elaborate image analyses. The frequency of erroneous chromosomal assignments depends on numerous factors, varying from experiment to experiment and from one observer to another. The influence of such "noise" however can be largely eliminated by appropriate statistics based on a sufficient number of evaluated CGH-metaphase spreads. According to our experience, chromosomal imbalances can even be detected by visual inspection of color diapositives of CGH-metaphase spreads taken with a conventional fluorescence microscope. In the latter case, exposure times have to be chosen for optimal assessment of fluorescence intensities in individual chromosomes. A cooled CCD camera, however, is highly advantageous, since simple thresholding procedures can be applied in order to introduce objective criteria for marking suspicious chromosomes. The following steps are recommended further to improve the assignment of chromosomes by visual examination. (1) In a given CGH-metaphase spread, chromosomes that are obviously painted to a greater or to a lesser degree than the majority are designated by an observer. (2) A threecolor look-up table is created choosing the threshold range 
with the highest and the lowest possible thresholds that exclude all designated chromosomes. Application of this threecolor look-up table may lead to the detection of additional chromosomes or chromosome segments that fall outside the chosen threshold range, but that have not yet been recognized by visual inspection. These additional chromosomes or segments are included as candidates for gains or losses. (3) Steps 1 and 2 are repeated. The number of metaphase spreads that need to be evaluated for a reliable diagnosis has to be established empirically. (4) Specific chromosomes or segments that are selected in this way with frequencies significantly higher than expected for a random assignment are considered as being over-represented or under-represented in the test genome. Automation of FR measurements in CGH-metaphase spreads provides a challenging task for future development, and could open the way for routine applications of CGH independent from any subjective interference.

Specificity and sensitivity of diagnoses in CGH experiments. The sensitivity and specificity of the CGH diagnosis depends on the choice of thresholds used to separate balanced from unbalanced chromosomes in the test genome. A compromise has to be chosen between sensitivity and specificity depending on the goals of the experiment and on the availability of independent methods to confirm the results (see below). Too restrictive thresholds, although increasing the specificity, may decrease the sensitivity of CGH diagnoses, enlarging the number of "false negatives", i.e., unbalanced chromosome types falsely classified as being balanced in the test genome. On the other hand, too permissive criteria could lead to a large number of "false" positives", i.e., chromosome types wrongly considered to be unbalanced in the test genome.

To detect chromosomal imbalances present in subclones of decreasing representation in the test sample, a more permissive threshold has to be chosen. The presence of normal cells in tumor tissues will also impair the sensitivity of $\mathrm{CGH}$, again suggesting the choice of a more permissive threshold. False positives could then be eliminated by independent tests with chromosome-specific DNA probes (see the integrated approach discussed below). On the other hand, if test DNA can be prepared after separation of tumor cells from normal cells, e.g., by flow sorting of suspended cells or by microdissection of solid tumor tissues (see below), a more restrictive threshold may be preferable.

The minimum size of chromosome material for which gains or losses can be detected by $\mathrm{CGH}$ is of major importance, but cannot be assessed clearly at the present time. In our present experiments, unbalanced chromosomal material equal to or greater than $40 \mathrm{Mbp}$ was unequivocally identified. We expect, however, that much smaller segments can be detected after further optimization of $\mathrm{CGH}$ and image analysis procedures (D. Pinkel, personal discussion).

\section{An integrated approach for chromosome analyses}

The tools that are now at hand for chromosome analysis range from procedures useful for the global screening of chromosomal changes to the analysis of individual DNA sequences. These tools need to be applied in a sequence that optimally fits the needs of each investigation. In such an integrated approach, the advantages of each method will comple- ment the limitations of the others. Wherever metaphase spreads from a clinical or tumor specimen are available, chromosome banding provides the method of choice for a comprehensive and rapid analysis of both balanced and unbalanced chromosome rearrangements at the single cell level. Its resolution, however, is limited and its results may not adequately reflect the clonal heterogeneity of the test specimen, particularly in cases where metaphase spreads are prepared after short or even long term culture. In cases where chromosome banding is not applicable or provides insufficient results, CGH can now be used as an additional global and rapid screening test to detect genetic imbalances predominant in a test specimen. FISH and molecular genetic approaches (see Introduction) provide the tools for confirming and studying specific chromosome aberrations suggested by the results of banding analyses and/or CGH with high resolution. A rapidly increasing number of chromosome-band-specific DNA probes can be chosen that optimally fit the needs of molecular cytogenetics (Bellané-Chantelot et al. 1992; Lengauer et al. 1992). Using interphase cytogenetics, representative samples of nuclei from both the original tumor specimen and from the corresponding cell culture can be analyzed in order to distinguish culture-dependent changes in the proportions of various clones. Recent developments of multiple color FISH (Ried et al. 1992) have greatly enhanced the usefulness of FISH as a diagnostic tool. The present study demonstrates that probes labeled with various proportions of biotin and digoxigenin can be used to enhance further the number of chromosome targets that can be distinguished by color. The finding that three different ranges of fluorescence ratios can be distinguished without overlap for combinations of two fluorochromes, suggests that four spectrally separable fluorochromes in various proportions may suffice to distinguish all chromosomes of the human chromosome complement by fluorescence ratio measurements.

\section{Perspectives}

New diagnostic and research scenarios can be envisaged using $\mathrm{CGH}$. Some examples are briefly considered below to illuminate this potential.

(1) CGH can be applied to study differences between genomes of related species and also differences between individuals of the same species. For example, chromosomespecific low-abundance repetitive sequences have been reported to occur over a significant portion of chromosome 16 (Dauwerse et al. 1992; Stallings et al. 1992). Polymorphisms of such low abundance repeats might become detectable by CGH. Since, in the present experiments, tumor test DNA and control DNA were used from different individuals, a polymorphism of such sequences may be considered as a possible explanation for the discrepancies concerning chromosome 16 observed in the T-PLL case between the results of chromosome banding and CGH analysis.

(2) CGH should become of great importance in identifying genetic imbalances in patients considered suspicious for a chromosomal syndrome. Banding analyses have often failed to identify the origin of small unbalanced segments of chromosomes, in particular in patients with de novo rearrangements. Candidate chromosome regions suspicious for a gain or loss of genetic material identified by CGH can be mapped 
in detail using appropriate sets of DNA probes in combination with multiple color FISH to metaphase spreads from the patient (unpublished experiments). Such a combined approach could dramatically improve karyotype/phenotype comparisons.

(3) Degenerate oligonucleotide primers (DOP) have recently been developed to amplify uniformly minute amounts of DNA in PCR assays (Telenius et al. 1992). DOP-PCR amplified test DNA has been successfully applied as a probe in $\mathrm{CGH}$ experiments (own unpublished observations). The demands on the structural integrity of isolated DNA and the size of amplification products are low, since the average length of genomic DNA fragments required for $\mathrm{CGH}$ can be as small as $100 \mathrm{bp}$. DOP-PCR should become a highly useful technique for amplifying test DNA for CGH analyses from selected cell areas, microdissected from tissue sections. Considering the high sensitivity of this method previously reported for DNA amplification of microdissected chromosome materials (Meltzer et al. 1992), it might become possible to amplify DNA useful for CGH analyses even from single cells. We expect that CGH will open the way to studying chromosome imbalances in archived paraffin-embedded tissue sections from many solid tumors that were previously not available for cytogenetic analyses. $\mathrm{CGH}$ should facilitate the comparative analysis of chromosomal imbalances in histologically similar tumors from different patients, and comparative cytogenetic analyses of tumors from human and animals (e.g., mouse, rat). In cases where the genetic mechanisms involved in the development of histologically similar tumor types occurring in different species are similar, one would expect that chromosome consensus regions consistently involved in gains and losses should be detected that contain homologous genes important for the tumor type in question. In contrast, involvement of distinctly non-homologous chromosome regions would indicate profound differences in the genetic mechanisms. The general applicability of such an approach depends on detailed genetic maps for the species in question.

We expect that the new possibilities for a comprehensive and rapid mapping of genetic imbalances in tumor genomes (see also Joos et al. 1992) will help in the search for oncogenes and suppressor genes specifically involved in certain tumors (Weinberg 1991) and will improve classification schemes. Finally, it is hoped that a combination of CGH and other tools of molecular genetics and cytogenetics will become useful in the future in rapidly identifying such genes involved in tumors from individual patients. Such improved diagnostic schemes could pave the way for individually designated therapies based on the suppression of harmful gene actions or the restoration of those that are desired.

Acknowledgements. We thank Patricia Emmerich-Bock, Thomas Fink, Anette Kurz, and Thomas Ried for help in the evaluation of CGH experiments by visual inspection, Brigitte Schoell for expert technical assistance, and Angelika Wiegenstein for photographic work. We also thank G. Brugal and C. Cremer for stimulating discussions on fluorescence ratio imaging. This work was supported by grants from the Deutsche Krebshilfe (W23/90/Cr), the Commission of the European Communities (CT910029) and the Land Baden Württemberg (Förderung von Forschungsschwerpunkten an den Universitäten). S.d.M. was supported by stipends of the "Region Rhônes-Alpes" and the Commission of European Communities in the framework of Human Genome Analysis (GENO-913003).

\section{References}

Aikens RS, Agard DA, Sedat JW (1989) Solid-state imagers for microscopy. Methods Cell Biol 29:291-313

Bellané-Chantelot C, Lacroix B, Ougen P, Billault A, Beaufils S, Bertrand S, Georges I, Gilbert F, Gros I, Lucotte G, Susini L, Codani JJ, Gesnouin P, Pook S, Vaysseix G, Lu-Kuo J, Ried T, Ward D, Chumaskov I, Le Paslier D, Barrillot E, Cohen D (1992) Mapping the whole human genome by fingerprinting yeast artificial chromosomes. Cell 70:1059-1068

Bishop JM (1987) The molecular genetics of cancer. Science 235: 305-311

Bright GR, Fisher GW, Rogowska J, Taylor DL (1989) Fluorescence ratio imaging microscopy. Methods Cell Biol 30: 157-192

Caspersson T, Farber S, Foley GE, Kudynowski J, Modest EJ, Simonsson E, Wagh U, Zech L (1968) Chemical differentiation along metaphase chromosomes. Exp Cell Res 49:219-226

Collins C, Kuo WL, Segraves R, Fuscoe J, Pinkel D, Gray J (1991) Construction and characterization of plasmid libraries enriched in sequences from single human chromosomes. Genomics 11:997-1006

Dauwerse JG, Jumelet EA, Wessels JW, Saris JJ, Hagemeijer G, Beverstock G, Ommen GJB van, Breuning MH (1992) Extensive cross-homology between chromosome 16p and 16q may explain inversions and translocations. Blood (in press)

Hiraoka Y, Paddy MR, Swedlow JR, Agard DA, Sedat JW (1991) Three-dimensional multiple wavelength microscopy for the structural analysis of biological phenomena. Semin Cell Biol 2: 153-165

Humbert C, Santisteban MS, Usson Y, Robert-Nicoud M (1992) Intranuclear co-localization of newly replicated DNA and PCNA by simultaneous immunofluorescent labelling and confocal microscopy in MCF-7 cells. J Cell Sci 103:97-103

Jauch A, Daumer C, Lichter P, Murken J, Schroeder-Kurth T, Cremer T (1990) Chromosomal in situ suppression hybridization of human gonosomes and autosomes and its use in clinical cytogenetics. Hum Genet 85:145-150

Joos S, Falk MH, Lichter P, Haluska FG, Henglein B, Lenoir GM, Bornkamm GW (1992) Variable breakpoints in Burkitt lymphoma cells with chromosomal $t(8 ; 14)$ translocations separate $c$-myc and the IgH locus up to several hundred $\mathrm{kb}$. Hum Mol Genet 1:625-632

Joos S, Scherthan H, Speicher MR, Schlegel J, Cremer T, Lichter P (1993) Detection of amplified DNA sequences by reverse chromosome painting using genomic tumor DNA as probe. Hum Genet 90 : $584-589$

Kallioniemi O-P, Kallioniemi D, Rutovitz D, Sudar D, Gray JW, Waldeman F, Pinkel D (1992) Comparative genomic hybridization: a new method based on isolated DNA to determine gains and losses of DNA sequences anywhere in the genome in a single hybridization (abstract). Am J Hum Genet 51: A23

Kaplan KB, Sweldow JR, Varmus HE, Morgan DO (1992) Association of p60 6 -sic with endosomal membranes in mammalian fibroblasts. $J$ Cell Biol 118:321-333

Koenig M, Moisan JP, Heilig R, Mandel JL (1985) Homologies between $\mathrm{X}$ and $\mathrm{Y}$ chromosomes detected by DNA probes: localisation and evolution. Nucleic Acid Res 13:5485-5501

Kovacs G, Fuzesi L, Emanuel A, Kung H (1991) Cytogenetics of papillary cell tumors. Genes Chromosomes Cancer 3:239-255

Lange JHM de, Schipper NW, Schuurhuis GJ, Kate TK ten, Heijningen THM van, Pinedo HM, Lankelma J, Baak JPA (1992) Quantification by laser scan microscopy of intracellular doxorubicin distribution. Cytometry 13:57I-576

Lengauer C, Riethman HC, Speicher MR, Taniwaki M, Konecki D, Green ED, Becher R, Olson MV, Cremer T (1992) Metaphase and interphase cytogenetics with Alu-PCR amplified YAC clones containing the BCR-gene and the protooncogenes c-raf-I, c-fms, c-erbB-2. Cancer Res 52:2590-2596

Lichter P, Cremer T (1992) Chromosome analysis by non-isotopic in situ hybridization: In: Human cytogenetics: a practical approach. IRL, Oxford, pp 157-192

Lichter P, Cremer T, Borden J, Manuelidis L, Ward DC (1988) Delineation of individual human chromosomes in metaphase and interphase cells by in situ suppression hybridization using recombinant DNA libraries. Hum Genet 80:224-234

Lichter P, Boyle AL, Cremer T, Ward DC (1991) Analysis of genes and chromosomes by non-isotopic in situ hybridization. Genet Anal Tech Appl 8:24-35 
Matutes E, Britto-Babapulle V, Swansbury J, Ellis J, Morilla R, Dearden C. Sempere A, Catovsky D (1991) Clinical and laboratory features of 78 cases of T-prolymphocytic leukemia. Blood 78:22693274

Meltzer PS, Guan XY, Burgess A, Trent JM (1992) Rapid generation of region specific probes by chromosome microdissection and their applications. Nature Genet $1: 24-28$

Nederlof PM (1991) Methods for quantitative and multiple in situ hybridization. Doctoral thesis, University of Leiden, The Netherlands

Nederlof PM, Flier S van der, Wiegant J, Raap AK, Tanke HJ, Ploem JS, Ploeg $M$ van der (1990) Multiple fluorescence in situ hybridization. Cytometry $11: 126-131$

Page DC, Mosher R, Simpson EM, Fisher EMC, Mardon G, Pollack J, McGillivray B, Chapelle A de la, Brown LG (1987) The sex-determining region of the human $\mathrm{Y}$ chromosomes encodes a finger protein. Cell 51:1091-1104

Pinkel D, Straume T, Gray JW (1986) Cytogenetic analysis using quantitative, high sensitivity, fluorescence hybridization. Proc Natl Acad Sci USA 83:2934-2938

Pinkel D, Landegent J, Collins C, Fuscoe J, Segraves R, Lucas J, Gray JW (1988) Fluorescence in situ hybridization with human chromosome-specific libraries: detection of trisomy 21 and translocations of chromosome 4. Proc Natl Acad Sci USA 85:9138-9142

Poddighe PJR, Ramaekers FCS, Hopman AHN (1992) Interphase cytogenetics of tumors. J Pathol 166:215-224

Note added in proof. The following important papers have appeared in print since submission of this paper: Nederlof PM, van der Flier S, Vrolijk J, Tanke HJ, Raap AK (1992) Fluorescence ratio measurements of double-labeled probes for multiple in situ hybridization by digital imaging microscopy. Cytometry 13:839-845; Kallioniemi A, Kallioniemi O-P, Sudar D, Rutovitz D, Gray JW, Waldman F, Pinkel D (1992) Comparative genomic hybridization for molecular cytogenetic analysis of solid tumors. Science $258: 818-82$ ]
Ried T, Baldini A, Rand TC, Ward DC (1992) Simultaneous visualization of seven different DNA probes by in situ hybridization using combinatorial fluorescence and digital imaging microscopy. Proc Natl Acad Sci USA 89:1388-1392

Robert-Nicoud R, Arndt-Jovin DJ, Schormann TTJ (1989) 3-D imaging of cells and tissues using confocal laser scanning microscopy and digital processing. Eur J Cell Biol 4 [Suppl 25] : 49-52

Stallings RL, Dogett NA, Okumura K. Ward DC (1992) Chromosome 16-specific repetitive DNA sequences that map to chromosomal regions known to undergo breakage/rearrangement in leukemia cells. Genomics 13:332-338

Telenius H, Pelmear AH, Tunnacliffe A, Carter NP, Behmel A, Ferguson-Smith MA, Nordenskjöld M, Pfragner R, Ponder BAJ (1992) Cytogenetic analysis by chromosome painting using DOP-PCR amplified flow-sorted chromosomes. Genes Chromosomes Cancer 4: $257-263$

Usson Y, Torch S, Drouet d'Aubigny G (1987) A method for automatic classification of large and small myelinated fibre populations in peripheral nerves. J Neurosci Methods 20:237-248

Waggoner A, Debasio R, Conrad P, Bright GR, Ernst L, Ryan K. Nederlof M, Taylor D (1989) Multiple spectral parameter imaging. Methods Cell Biol 30:449-478

Weinberg RA (1991) Tumor suppressor genes. Science 254: 1138-1146 